\title{
Dänisch oder Deutsch? Die Ergebnisse einer Fragebogenaktion unter norddeutschen und dänischen Manager_innen zum Sprach- gebrauch und -bedarf ihrer Unternehmen
}

Sonja Vandermeeren (Kiel)

\begin{abstract}
The present article first defines the terms language choice, the need for foreign language competence and the need for knowledge about foreign cultures. Then, attention is focused on empirical material, which is discussed against the background of these terms. In order to gather the data dealt with in this article, German and Danish (hotel) managers were presented a questionnaire. Taking the case of German companies/hotels which are in regular contact with Danes, the questionnaire investigates their need for Danish and whether knowledge about Danish culture is required of employees. The German managers were also asked about the frequency of German, Danish and English language use by their employees and about their own language behavior when communicating with speakers of Danish. Also data on Danish language knowledge and knowledge about Danish culture are collected. The present article compares the results of the survey in Germany with the results which were obtained in Denmark: Which need for German and for knowledge of German culture do Danish managers perceive?/How do they rate their own proficiency in German and that of their employees?/How often do they choose German, English and Danish in communication with Germans?
\end{abstract}

\section{$1 \quad$ Einleitung}

Das Englische erfüllt einen wichtigen, pragmatischen Zweck in der internationalen Kommunikation. Die Verwendung anderer Sprachen ist jedoch auch erforderlich. Vor allem in der internationalen Wirtschaftskommunikation kann sich das Nichtverwenden der Landessprache der Geschäftspartner_innen zu einem Störfaktor in zwischenbetrieblichen Beziehungen entwickeln.

Der vorliegende Beitrag vermittelt sowohl ein Gesamtbild der Sprachwahl deutscher Firmen und Hotels in der Kommunikation mit Dänen als auch ein Gesamtbild der Sprachwahl dänischer Firmen und Hotels in der Kommunikation mit Deutschen. Für die Zustandsbeschreibung des Fremdsprachengebrauchs von Unternehmen lassen sich in erster Linie zwei Faktoren heranziehen: Fremdsprachenbedarf und Fremdsprachenkenntnisse. Es wird versucht, die Dänischkenntnisse, den Gebrauch der dänischen, deutschen und englischen Sprache sowie den Bedarf an Dänischkenntnissen und den Bedarf an Wissen um die dänische Kultur deutscher Hotels und Betriebe zu quantifizieren. Parallel dazu werden die Deutschkenntnisse und

Linguistik online 79, 5/2016 - http://dx.doi.org/10.13092/lo.79.3347

CC by 3.0 
der Deutsch-, Dänisch- und Englischgebrauch dänischer Hotels/Firmen sowie ihr Bedarf an Deutschkenntnissen und an Wissen über die deutsche Mentalität beschrieben. Die Forschungsergebnisse entstanden aus dem mit Mitteln des Europäischen Fonds für regionale Entwicklung (INTERREG4A Syddanmark-Schleswig K.E.R.N) finanzierten Projekt Nationale Stereotype und Marketingstrategien in der interkulturellen deutsch-dänischen Kommunikation (kurz SMiK). Die Verfasserin bearbeitete das Teilprojekt Interkulturelle Kommunikation, Kulturdimensionen und Stereotype eigenverantwortlich. Im Rahmen dieses Teilprojekts wurde eine breit angelegte Umfrage unter norddeutschen und dänischen Firmenmanager_innen bzw. Hoteldirektor_innen durchgeführt.

Bevor die Ergebnisse dieser Fragebogenaktion präsentiert werden, wird ein theoretischer Unterbau geliefert. Auf die Beschreibung der Methodik folgt die statistische Analyse mit der Interpretation der gewonnenen Daten im deutsch-dänischen Vergleich. Abschließend werden die Ergebnisse zusammengefasst und deren Konsequenzen reflektiert.

\section{Begriffserläuterungen}

\subsection{Adaptation, Standardisierung und Nicht-Adaptation}

Ich bezeichne die Strategie eines Unternehmens, für die Kommunikation mit einem Kommunikationspartner dessen Muttersprache zu wählen, als Adaptation. Die Strategie, die in der Diskussion um internationales Marketing neben der Adaptation eine große Rolle spielt, ist die der Standardisierung - des Produkts, der Marke, des Preises, der Qualität, der Werbung und des Sprachgebrauchs. Mit Standardisierung bezeichne ich die systematische Verwendung der englischen Sprache als Lingua franca in der internationalen Kommunikation durch Nichtenglischsprachige. Die Verwendung der eigenen Landessprache in internationalen Kontakten kann dann Nicht-Adaptation genannt werden (Vandermeeren 1998: 36-38).

Ist die gewählte Fremdsprache die Muttersprache des Kommunikationspartners, so wird diese Sprachwahl Adaptation genannt. Ich bin mir aber durchaus bewusst, dass kommunikatives Verhalten in einer Fremdsprache gegenüber einem Gesprächspartner, für den diese Sprache Muttersprache ist, auch von einer in dessen Kulturkreis üblichen Kommunikationsmentalität begleitet werden muss, um Anklang zu finden. Dass Fremdsprachengebrauch ohne kulturelle Anpassung nicht genügt, wird vorausgesetzt. Der Hauptakzent liegt aber im vorliegenden Beitrag auf quantitativen Aspekten des Fremdsprachengebrauchs.

Sprache und Kultur werden in diesem Beitrag analytisch auseinandergehalten, obwohl sie, wie gerade erwähnt, sehr eng miteinander verknüpft sind. Diese Trennung ist sinnvoll, weil kulturelle Anpassung nicht immer mit sprachlicher Anpassung einhergeht. Bei sprachlicher Adaptation ist jedoch in der Regel mindestens ein minimales Wissen um die fremde Kultur vorhanden.

\subsection{Fremdsprachenbedarf}

Ich gehe - wie die meisten Forscher_innen - davon aus, dass für Betriebe mit regelmäßigen Geschäftsverbindungen zu einem bestimmten Land ein unmittelbarer Anlass besteht, die Sprache dieses Landes zu verwenden. Der Fremdsprachenbedarf von Unternehmen ist in erster Linie durch den Export bedingt. Im Kontakt mit Geschäftspartner_innen und Kund_innen 
entsteht ein Bedarf aus (verkaufs)psychologischen Gründen. Rehbein (1995: 97f.) betont, dass derjenige, der die Landessprache seiner Geschäftspartner_innen beherrsche, am besten ausgestattet sei, um die eigenen Interessen $\mathrm{zu}$ fördern. Es ist seiner Meinung nach von allerhöchster Wichtigkeit, dass die Bedürfnisse des Gegenübers erfolgreich in eine (Kauf)Entscheidung umgesetzt werden. Der Entscheidungsprozess besteht größtenteils aus mentalen Änderungen. Aus psycholinguistischer Sicht finden viele mentale Prozesse, besonders diejenigen, die mit Motivationsmechanismen in Zusammenhang stehen, in der Muttersprache statt. Mit Motivationsmechanismen haben wir es ja hier zu tun, weil Bedürfnisse in einen Entschluss umgesetzt werden müssen.

$\mathrm{Zu}$ erwähnen sind noch weitere Gründe, die dafür sprechen, im wirtschaftlichen Bereich die Muttersprache des Gegenübers zu sprechen. Adaption ist nicht nur vorteilhaft für den Sprecher, dessen Muttersprache verwendet wird, sondern unter Umständen auch für den NichtMuttersprachler. Ersterer hat natürlich den Vorteil, sich in der Sprache, die er am besten kennt, ausdrücken zu können, aber letzterer hat dafür bei entsprechend großer linguistischer, kommunikativer und interkultureller Kompetenz die Gewissheit, dass sein Gesprächspartner ihn einwandfrei versteht, d. h., dass keine sprach- oder kulturbedingten Missverständnisse aufkommen. Coulmas (1987: 100) spricht in dem Zusammenhang vom „Kommunikationskontrollprinzip“. Der Nicht-Muttersprachler hat sozusagen die Kontrolle darüber, wieviel sein Gesprächspartner versteht. Im Dienstleistungssektor (z. B. im Hotelgewerbe) ist es so, dass man den Kunden/Gästen den allerbesten Service anbieten will. In der Face-to-FaceInteraktion ihre Sprache zu verwenden, erhöht deren Zufriedenheit.

Es wird häufig Fremdsprachenbedarf mit einem seiner Indikatoren, nämlich Fremdsprachengebrauch, vermischt. Diese Vermischung ist verständlich. In der Regel wird Fremdsprachenbedarf mit Hilfe des Begriffs Sprachgebrauch erklärt. Ich habe versucht, eine disambiguierende, soziolinguistische Definition für betrieblichen Fremdsprachenbedarf zu formulieren, die es zulässt, die Begriffe Fremdsprachenbedarf und Fremdsprachengebrauch voneinander zu trennen und sie dennoch miteinander in Zusammenhang zu bringen: Der Fremdsprachenbedarf von Unternehmen ist ihr Bedarf an Mitarbeiter_innen, die zusammen eine ausreichend große Anzahl von Fremdsprachen in ausreichendem Maße beherrschen, um geschäftliche Kontakte mit Anderssprachigen in deren Muttersprache oder in einer Lingua franca anknüpfen und pflegen zu können. Unternehmen haben einen Mehrbedarf an Fremdsprachen, wenn die kommunikative Kompetenz ihrer Mitarbeiter_innen ihren Bedarf an Fremdsprachen nicht deckt. Der tatsächliche Fremdsprachengebrauch eines Unternehmens kann nur dann als mit seinem Fremdsprachenbedarf identisch betrachtet werden, wenn kein Mehrbedarf an Fremdsprachen vorausgesetzt wird (cf. Vandermeeren 1998: 60).

Äußerungen über Fremdsprachenbedarf sind subjektiv und daher konfliktanfällig. Die Behauptung, dass ein Unternehmen Fremdsprachen benötige, heißt aufgrund bestimmter Einstellungen Fremdsprachengebrauch als erforderlich $\mathrm{zu}$ betrachten. Es ist wichtig, dass Forscher_innen, die Bedarfsanalysen mittels Befragungen durchführen, sich dessen bewusst sind, dass die Frage nach dem Fremdsprachenbedarf, den die Informant_innen sehen, deren Einstellungen zum Fremdsprachenbedarf erfassen. Auch die Einstellung der Forscher_innen ihre Meinung darüber, in welchem Ausmaß Unternehmen welche Fremdsprachen benötigen beeinflusst ihr Forschungsdesign und ihre Interpretation der Daten. 
Der Begriff Fremdsprachenbedarf wird in diesem Beitrag in Anlehnung an Vandermeeren (1998: 70-71), Vandermeeren (2003: 14) und Vandermeeren (2005: 161-163) mit Hilfe von vier verschiedenen Variablen operationalisiert:

- von der Frequenz der Kontakte mit Deutschen bzw. Dänen,

- von den Meinungen der befragten Manager_innen bezüglich des Deutsch- bzw. Dänischbedarfs ihrer Unternehmen,

- von den in ihren Unternehmen vorhandenen Deutsch- bzw. Dänischkenntnissen,

- von der Häufigkeit, mit der ihre Firmen/Hotels die deutsche bzw. dänische Sprache benutzen.

Diese Variablen ergeben sich aus den folgenden Fragen:

1. Wie oft hat Ihr Unternehmen direkte Geschäftskontakte mit Deutschen bzw. Dänen?

2. Wie oft wird Ihr Unternehmen die nächsten Jahre Deutsch- bzw. Dänischkenntnisse benötigen?

3. Sind Ihres Wissens nach genug Deutsch- bzw. Dänischkenntnisse in Ihrer Firma/in Ihrem Hotel vorhanden?

4. Wie oft verwendet Ihr Unternehmen die folgenden Sprachen bei Kontakten mit Deutschen bzw. Dänen?

In den Antworten auf die ersten zwei Fragen spiegeln sich zwei Arten von Bedarf wider. Zum einen zeigt sich der objektive Fremdsprachenbedarf, wenn die Antworten auf die Frage nach der Frequenz der Kontakte mit ausländischen Firmen betrachtet werden (objektiv ist nicht der Fremdsprachenbedarf, sondern dessen Indikator: die Frequenz der Kontakte). Zum anderen präsentiert sich der subjektive Fremdsprachenbedarf, wenn die Meinungen der Befragten zum Fremdsprachenbedarf berücksichtigt werden (die Meinung von Befragten ist ein subjektiver Indikator). Indem die Antworten auf die erste Frage als Filter benutzt werden, ergeben sich drei neue Arten des Fremdsprachenbedarfs:

unbewusster Fremdsprachenbedarf, objektiver Fremdsprachenmehrbedarf und subjektiver Fremdsprachenmehrbedarf (in den untenstehenden Tabellen als unbewusst. FSB, obj. FSMB bzw. subj. FSMB abgekürzt).

Dänische Firmen/Hotels mit regelmäßigen/sehr häufigen Kontakten zu Deutschen beispielsweise, die angeben, dass es nie/nur selten nötig für sie sei, Deutsch zu benutzen, haben einen unbewussten Deutschbedarf ( $=b-F e l d ; b+d=100 \%$ ). 


\begin{tabular}{|l|l|l|l|}
\cline { 3 - 3 } \multicolumn{2}{c|}{} & \multicolumn{2}{l|}{$\begin{array}{l}\text { Objektiver Fremdsprachenbedarf } \\
\text { (Filter) }\end{array}$} \\
\cline { 3 - 4 } \multicolumn{2}{l|}{} & nie/selten & regelmäßig/ sehr oft \\
\hline \multirow{2}{*}{ Subjektiver Fremdsprachenbedarf } & nie/selten & a & b $=$ unbewusst. FSB \\
\cline { 2 - 4 } & regelmäßig & c & d \\
\hline
\end{tabular}

Tabelle 1: Unbewusster Fremdsprachenbedarf (unbewusst. FSB)

Noch ein Beispiel: Deutsche Firmen mit regelmäßigen bis sehr häufigen Kontakten zu Dänen, die angeben, dass sie nie/nur selten Dänisch benutzen, haben einen objektiven Dänischmehrbedarf.

\begin{tabular}{|c|c|c|c|}
\hline & & \multicolumn{2}{|c|}{$\begin{array}{l}\text { Objektiver Fremdsprachenbedarf } \\
\text { (Filter) }\end{array}$} \\
\hline & & nie/selten & regelmäßig/ sehr oft \\
\hline \multirow[b]{2}{*}{ Fremdsprachengebrauch } & nie/selten & $\mathrm{a}$ & $b=$ obj. FSMB \\
\hline & $\begin{array}{l}\text { regelmäßig/ } \\
\text { (fast) immer }\end{array}$ & c & d \\
\hline
\end{tabular}

Tabelle 2: Objektiver Fremdsprachenmehrbedarf (obj. FSMB)

Bei Unternehmen, die einen regelmäßigen bis sehr häufigen objektiven Fremdsprachenbedarf sowie einen subjektiven Bedarf an Fremdsprachenkenntnissen haben und angeben, dass sie über keine oder zu wenig Fremdsprachenkenntnisse verfügen, lässt sich ein subjektiver Fremdsprachenmehrbedarf feststellen.

\begin{tabular}{|c|c|c|c|}
\hline & & \multicolumn{2}{|c|}{$\begin{array}{l}\text { Objektiver Fremdsprachenbedarf } \\
\text { (Filter) }\end{array}$} \\
\hline & & nie/selten & regelmäßig/ sehr oft \\
\hline \multirow{2}{*}{$\begin{array}{l}\text { Fremdsprachenkenntnisse } \\
\text { (ein subjektiver Bedarf an Fremd- } \\
\text { sprachenkenntnissen ist vorhanden) }\end{array}$} & $\begin{array}{l}\text { keine oder zu } \\
\text { wenige }\end{array}$ & $\mathrm{a}$ & $\mathrm{b}=$ subj. FSMB \\
\hline & genügend & $\mathrm{c}$ & $\mathrm{d}$ \\
\hline
\end{tabular}

Tabelle 3: Subjektiver Fremdsprachenmehrbedarf (subj. FSMB)

\subsection{Bedarf an Kulturwissen}

Das Objekt des Fremdsprachenbedarfs sind nicht nur Kenntnisse bezüglich Aussprache, Grammatik und Lexikon, sondern die gesamten kulturgebundenen kommunikativen Fähigkeiten. Beamer (1992: 285-287) betont, dass man sich der interkulturellen kommunikativen 
Kompetenz am besten ausgehend von einem Kommunikationsmodell nähern kann. Der Empfänger hantiert mit Werten und kulturellen Variablen, die dem Sender der Botschaft möglicherweise unbekannt sind. Das heißt, dass kognitiv erworbenes Wissen über Kulturen die Basis für die Entwicklung einer interkulturellen kommunikativen Kompetenz ist.

In ihrem Buch „Interkulturelle Wettbewerbsstrategien“ unterscheiden Scholz/Stein (2013) drei Arten des traditionellen betrieblichen Kulturmanagements (das Chamäleon-Verhalten, das Cowboy-Verhalten und das Kultur-Nivellieren) und plädieren für ein neues, positivistisches Denken als Alternative. Das Kultur-Chamäleon hat als zentrale Handlungsstrategie: „Assimiliere dich kulturell in der jeweiligen Umgebung. [...] [Die Assimilation] beginnt mit der Sprache, [...] erreicht irgendwann die Sitten und Gebräuche, [...] um bei den Hintergründen zu Land und Leuten, [...] lange noch nicht aufzuhören. Welch ein Lernprogramm bereits schon für ein einziges Land. [...] Sich in Gänze anzupassen ist [also] nicht möglich“ (Scholz/Stein 2013: 29f.). Die Handlungsmaxime des Kultur-Cowboys lautet: „,verhalte dich nicht anders als gewohnt" (Scholz/Stein 2013: 45), während der Kultur-Nivellierer in allen Ländern das Gleiche tut. Die Grundannahme des Kultur-Nivellierers ist, dass es universelle Normen gibt, die für alle Kulturen passen (Scholz/Stein 2013: 51). Scholz und Stein sind der Meinung, dass ein gutes Kulturmanagement darin besteht, genau zu differenzieren, wo eine Anpassung notwendig ist und wo nicht (Scholz/Stein 2013: 65). Sowohl keine Anpassung als auch eine sehr große Anpassung sind Störfaktoren. Eine gemäßigte Anpassung dagegen wirkt sich günstig auf den Gesprächsablauf aus.

Eine gemäßigte Anpassung impliziert, dass Gesprächspartner_innen ,aushandeln“, welche Normen für die Dauer des Gesprächs gelten sollen. Laut Beneke (1991: 60) führen Gesprächspartner_innen auf diese Weise eine temporäre soziokulturelle Konvergenz herbei.

Der Bedarf an Wissen um Fremdkulturen kann - ähnlich wie der Bedarf an Fremdsprachenkenntnissen - operationalisiert werden. Da sich Informant_innen anders als bei ihren Sprachwahlstrategien nicht immer dessen bewusst sind, ob sie Strategien der kulturellen Sensibilisierung einsetzen und welche dies sind, konnte der Bedarf an Kulturkenntnissen im Rahmen der Fragebogenaktion in Deutschland und Dänemark nur mit Hilfe von drei Variablen operationalisiert werden.

Die dazugehörigen Fragen lauten:

1. Wie oft hat Ihr Unternehmen Kontakte mit Deutschen bzw. Dänen?

2. Wie viel Wissen über die deutsche bzw. dänische Mentalität benötigt Ihr Unternehmen für den Kontakt mit Dänen bzw. Deutschen?

3. Ist Ihrer Meinung nach genügend Wissen um die deutsche bzw. dänische Mentalität in Ihrem Unternehmen vorhanden?

Die Antworten auf die erste Frage quantifizieren den objektiven Bedarf an Kulturwissen, die Antworten auf die zweite Frage den subjektiven Bedarf an Kulturwissen. Wenn Frage 1 als Filterfrage benutzt wird, können zwei weitere Arten des Bedarfs an Wissen um Fremdkulturen erfasst werden: unbewusster Bedarf und subjektiver Mehrbedarf an Fremdkulturwissen.

Das folgende Beispiel demonstriert den unbewussten Bedarf an Fremdkulturwissen. Ein dänisches Unternehmen mit regelmäßigen/sehr häufigen Geschäftskontakten zu Deutschland, deren Manager_innen angeben, dass es kein/sehr wenig Wissen um die deutsche Mentalität be- 
nötigt, hat einen unbewussten Bedarf an Wissen um die deutsche Kultur (=b-Feld; $\mathrm{b}+\mathrm{d}=100 \%)$.

\begin{tabular}{|c|c|c|c|}
\hline & & \multicolumn{2}{|c|}{$\begin{array}{l}\text { Objektiver Bedarf an Fremdkultur- } \\
\text { wissen (Filter) }\end{array}$} \\
\hline & & nie/selten & regelmäßig/ sehr oft \\
\hline \multirow{2}{*}{$\begin{array}{l}\text { Subjektiver Bedarf an Fremdkultur- } \\
\text { wissen }\end{array}$} & nie/selten & $\mathrm{a}$ & $\begin{array}{l}\mathrm{b}=\text { unbewusst. } \\
\text { FKWB }\end{array}$ \\
\hline & $\begin{array}{l}\text { regelmäßig/ } \\
\text { sehr oft }\end{array}$ & $\mathrm{c}$ & d \\
\hline
\end{tabular}

Tabelle 4: Unbewusster Bedarf an Fremdkulturwissen (unbewusst. FKWB)

Unternehmen, die einen regelmäßigen bis sehr häufigen objektiven sowie einen subjektiven Bedarf an Fremdkulturwissen haben und angeben, dass sie zu wenig Fremdkulturkenntnisse haben, zeigen einen subjektiven Mehrbedarf an Fremdkulturwissen.

\begin{tabular}{|l|l|l|l|}
\cline { 3 - 4 } \multicolumn{2}{l|}{} & \multicolumn{2}{l|}{$\begin{array}{l}\text { Objektiver Bedarf an Fremdkultur- } \\
\text { wissen (Filter) }\end{array}$} \\
\cline { 2 - 5 } & \multicolumn{2}{l|}{ nie/selten } & regelmäßig/ sehr oft \\
\hline $\begin{array}{l}\text { Fremdkulturkenntnisse } \\
\text { (ein subjektiver Bedarf an Fremdkul- } \\
\text { turkennrnissen ist vorhanden) }\end{array}$ & $\begin{array}{l}\text { keine oder zu } \\
\text { wenige }\end{array}$ & a & b $=$ subj. FKWMB \\
\cline { 2 - 5 } & genügend & c & d \\
\hline
\end{tabular}

Tabelle 5: Subjektiver Mehrbedarf an Fremdkulturwissen (subj. FKWMB)

Das Konzept Fremdkulturwissen wird in diesem Beitrag als Wissen um die Mentalität der Mitglieder der Fremdkultur operationalisiert. Theoretisch gesehen ist Kulturwissen viel weiter zu fassen als Wissen um Mentalität. Eine breiter gefächerte Operationalisierung würde auch Wissen um deutsche Unternehmenskultur (zum Beispiel Verhandlungstechniken) und Organisation deutscher Unternehmen (zum Beispiel Unternehmenshierarchie) einschließen, um nur einige Beispiele zu nennen (cf. Vandermeeren 2005: 173). In der vorliegenden Untersuchung mit Zielpersonen aus sehr vielen Branchen musste auf Fragen nach branchenspezifischen Formen des Bedarfs an Fremdkulturwissen verzichtet werden.

Außerdem werden in diesem Artikel Kultur und Nation gleichgesetzt, obwohl dies strenggenommen nicht zulässig ist. Losche/Püttker (2009: 22) weisen darauf hin, dass die Klassifikation von Nationen mittels bestimmter kultureller Merkmale zu wenig Differenzierung zeigt. In Anlehnung an Hofstede (1991: 12) gehen sie aber davon aus, dass viele Staaten ein historisch entwickeltes Ganzes sind, selbst wenn sie aus unterschiedlichen Gruppen bestehen. 


\section{$3 \quad$ Methodik}

Die Hauptuntersuchungsgegenstände Deutsch- bzw. Dänischgebrauch und Bedarf an Deutsch- bzw. Dänischkenntnissen wurden in Fragebogenvariablen zerlegt. Im Zuge der Operationalisierung der Untersuchungsobjekte wurden die Variablen in geschlossene Fragen umgewandelt. Um die Leistungen der Fragebögen zu prüfen, wurden die Fragebögen einem Prätest in Deutschland unterzogen. Die befragten Zielpersonen wurden auch gebeten, sich zur Fragebogengestaltung zu äußern. Dieser Prätest brachte unentbehrliche Daten über die Befragungssituation und die Qualität der Fragen hervor. Dem Inhalt nach haben sich die meisten Fragen im Prätest bewährt und die Fragen wurden von den Befragten nicht als zu schwierig empfunden. Außerdem überforderte die Anzahl der Fragen sie nicht.

Zusammenfassend lässt sich sagen, dass die Eindeutigkeit, Akzeptanz und Verständlichkeit der Fragebögen in befriedigendem Maße geprüft worden sind. Die Fragebögen liegen in zwei Versionen vor: eine deutschsprachige (für deutsche Informant_innen) und eine dänischsprachige (für dänische Informant_innen). Die Übersetzungen wurden von einer zweisprachigen Mitarbeiterin des überdachenden Projekts Nationale Stereotype und Marketingstrategien in der interkulturellen deutsch-dänischen Kommunikation angefertigt.

Der Schwerpunkt der vorliegenden Befragungsstudie liegt in der Empirie. Zentral ist eine computergestützte Analyse der gesammelten Antworten der verschiedenen Zielgruppen. Die statistischen Berechnungen wurden mit Hilfe des PC-Programms SPSS (Statistical Package for the Social Sciences) durchgeführt. Für jede Variable liegt eine eindimensionale Grundauszählung (Häufigkeitstabelle) vor. Eine fehlende Beobachtung (keine Angabe) wurde kein Wert zugewiesen, damit das Programm diesen Fall nicht mit in die Prozentberechnung einbezieht. Filterfragen sorgen dafür, dass der Code ,trifft nicht $\mathrm{zu}^{\text {“ }}$ in den Häufigkeitstabellen steht. Eine Filterfrage, zum Beispiel nach der Frequenz der Geschäftsbeziehungen mit deutschen Firmen, wurde formuliert, damit - sollte diese Frequenz „nie“ für eine befragte Firma sein - keine Angabe zum Sprachgebrauch mit deutschen Unternehmen von dieser Firma verlangt wird und später bei der SPSS-Auswertung der Code „trifft nicht zu“ in der Datenmatrixzeile dieser Firma für die Variable „Sprachgebrauch“ steht. Die Filterfrage nach den Kontakten mit Deutschen bzw. Dänen wird auch systematisch bei der Auswertung der Daten eingesetzt. Die Antworten der Hotels und Firmen, die nie oder selten Kontakte mit Deutschen bzw. Dänen haben, werden in diesem Beitrag in der Regel nicht berücksichtigt. Prozentzahlen werden nur für die Unternehmen, die regelmäßig bis sehr oft Kontakte mit Deutschen bzw. Dänen haben, berechnet, um die Aussagekraft der Daten zu steigern.

Weil die Stellung der dänischen Sprache in der deutschen Wirtschaft eher gering ist, wurde befürchtet, die deutschen Zielpersonen würden Fragebögen, die Dänischgebrauch als Thema haben, nicht ausfüllen. Ein Test sollte diesbezüglich Klarheit bringen: Vor der Durchführung der Umfragen wurde in Deutschland getestet, welche Rücklaufquote postalische und OnlineUmfragen im Vergleich erzielen und welche Hilfsmittel die Rücklaufquote erhöhen. Als Zielpersonen wurden Hotelmanager_innen genommen. Folgende Hilfsmittel wurden eingesetzt: Freiporto (ein beigefügter frankierter Antwortumschlag), das Versprechen, den Antwortenden die Befragungsergebnisse zukommen zu lassen und ein Erinnerungsschreiben/eine Erinnerungs-E-Mail. 
Aus den Ergebnissen des Tests geht hervor, dass eine Online-Fragebogenaktion in Deutschland mit einer Rücklaufquote von nur ca. $10 \%$ einhergeht, während eine postalische mehr als doppelt so viele deutsche Informant_innen rekrutiert. Außerdem zeigt sich, dass das Erinnerungsschreiben sich am besten als Verfahren zur Erhöhung der Rücklaufquote eignet.

Es wurde dennoch aus Kostengründen entschieden, die Hotels mittels eines OnlineFragebogens zu befragen. Die Firmen sollten aber postalisch befragt werden. Die Vollstichprobe der Hotels basierte auf allen Hoteleinträgen in den vier Bundesländern, die in Onlineportalen wie den Gelben Seiten und weiteren Internetverzeichnissen vorhanden sind. Die Online-Fragebogenaktionen in Deutschland und Dänemark fanden zwischen Kalenderwoche 47/2013 und Kalenderwoche 21/2014 statt. Die Hotels bekamen zusammen mit dem OnlineFragebogen auch einen Begleitbrief, in dem das Projekt kurz beschrieben wurde. In einem P. S. wurde das Versprechen gegeben, die Befragungsergebnisse auf Anfrage zuzuschicken. Jeweils ca. drei Wochen nach Absendung der E-Mails wurde eine Erinnerungsmail geschickt.

Die deutsche Firmenstichprobe basierte auf der Datenbank der Industrie- und Handelskammer für Unternehmen aller Branchen in Norddeutschland. Ausgewählt wurden nur Unternehmen, die Geschäftskontakte mit Dänemark haben. Auf dänischer Seite wurden die Firmen, die Geschäftsbeziehungen zu Deutschland unterhalten, online ausgewählt. Dazu wurde das Exportverzeichnis „ProDenmark“, das 3000 dänische Firmen (u. a. Exporteure) auflistet, benutzt. Die Umfrage unter Firmenmanagern wurde von Juli bis August 2014 postalisch durchgeführt und die Fragebogen und Begleitbriefe wurden namentlich an die Firmenmanager_innen geschickt. Die von deutschen Informant_innen ausgefüllten Fragebögen sollten an das deutsche SMiK-Büro und die dänischen an das dänische SMik-Büro zurückgesandt werden. Eine Erinnerungsaktion wurde von November 2014 bis Februar 2015 durchgeführt.

\section{Fragebogenaktion unter Hotelmanager_innen in Schleswig-Holstein, Hamburg, Niedersachsen und Berlin}

\subsection{Fragebogenvariable, Stichprobe, Rücklauf und fehlende Fälle}

Bei der Konstruktion des Fragebogens für deutsche und dänische Hotelmanager_innen wurden folgende Variable und Ausprägungen als Fragen beziehungsweise Antwortkategorien formuliert.

\section{Kontakte der Manager_innen mit deutschen bzw. dänischen Hotelgästen}

(nie, selten, regelmäßig, sehr oft)

\section{Deutsch-bzw. Dänischkenntnisse der Manager_innen}

(keine, bessere als geringe, nur geringe)

\section{Sprachgebrauch der Manager_innen}

Verwendungshäufigkeit der deutschen Sprache, der englischen Sprache und der dänischen Sprache im Kontakt mit deutschen bzw. dänischen Hotelgästen

(nie, selten, regelmäßig, (fast) immer)

4 Subjektiver Bedarf der Manager_innen an Kenntnissen der jeweiligen Fremdsprache 
(ja, nein)

5 Subjektiver Mehrbedarf der Manager_innen an Kenntnissen der jeweiligen Fremdsprache (ja, nein)

6 Wissen der Manager_innen an Wissen um die deutsche bzw. dänische Mentalität

(kein Wissen, sehr wenig, eher wenig, teils wenig/teils viel, eher viel, sehr viel)

7 Subjektiver Bedarf der Manager_innen an Wissen um die deutsche bzw. dänische Mentalität (ja, nein)

8 Subjektiver Mehrbedarf der Manager_innen an Wissen um die deutsche bzw. dänische Mentalität

(ja, nein)

9 Kontakte des Hotelpersonals mit deutschen bzw. dänischen Gästen

(nie, selten, regelmäßig, sehr oft)

10 Deutsch- bzw. Dänischkenntnisse des Hotelpersonals

10.1 Zahl der Mitarbeiter_innen mit Gästekontakt, die bessere als geringe Deutsch- bzw. Dänischkenntnisse haben

10.2 Zahl der Mitarbeiter_innen mit Gästekontakt, die nur geringe Deutsch- bzw. Dänischkenntnisse haben

10.3 Zahl der Mitarbeiter_innen mit Gästekontakt, die deutsche bzw. dänische E-Mails von Hotelgästen auf Deutsch bzw. Dänisch beantworten können

11 Sprachgebrauch des Hotelpersonals

Häufigkeit, mit der das Hotelpersonal die deutsche Sprache, die englische Sprache und die dänische Sprache im Kontakt mit deutschen bzw. dänischen Hotelgästen verwendet

(nie, selten, regelmäßig, (fast) immer)

12 Subjektiver Bedarf des Hotelpersonals an Kenntnissen der jeweiligen Fremdsprache

12.1 Jetziger Bedarf des Hotelpersonals mit Gästekontakt an Deutsch- bzw. Dänischkenntnissen

(ja, nein)

12.2 Zukünftiger Bedarf des Hotelpersonals mit Gästekontakt an Deutsch- bzw. Dänischkenntnissen

(überhaupt nicht, selten, regelmäßig, sehr oft)

13 Subjektiver Mehrbedarf des Hotelpersonals mit Gästekontakt an Kenntnissen der jeweiligen Fremdsprache

(ja, nein)

14 Wissen des Hotelpersonals mit Gästekontakt über die deutsche bzw. dänische Mentalität (eher nicht, eher ja, der Manager_in nicht bekannt) 


\section{Subjektiver Bedarf des Hotelpersonals an Fremdkulturwissen}

Bedarf des Hotelpersonals mit Gästekontakt an Wissen über die deutsche bzw. dänische Mentalität

(überhaupt nicht, sehr wenig, eher wenig, teils wenig/teils viel, eher viel, sehr viel)

\section{Subjektiver Mehrbedarf an Fremdkulturwissen}

Mehrbedarf des Hotelpersonals mit Gästekontakt an Wissen über die deutsche bzw. dänische Mentalität

(ja, nein)

Im Rahmen der Fragebogenaktion unter Hotelmanager_innen wurden, wie Tabelle 6 zeigt, 2407 deutsche Hoteldirektionen (davon 768 in Schleswig-Holstein, 366 in Hamburg, 714 in Niedersachen und 599 in Berlin) per E-Mail erreicht (bereinigte Stichprobe). Die Rücklaufquote in Deutschland beträgt nach zwei Erinnerungsmails $11 \%$. Von den 872 per E-Mail erreichten dänischen Hoteldirektionen füllten nach nur einer Erinnerungsmail 200 den OnlineFragebogen aus (Gesamtrücklaufquote 23,3 \%). Es gibt weniger Übernachtungen von Dänen in Norddeutschland als von Deutschen in Dänemark. So gab es im Jahr 2012 nur 2.855.677 Übernachtungen von dänischen Gästen in Deutschland (davon fielen 21,2 \% der Übernachtungen auf Berlin, 20,5\% auf Schleswig-Holstein, 8,3 \% auf Hamburg und 12,6\% auf Niedersachsen). In Dänemark übernachteten im Jahr 2012 insgesamt 21.681.067 Deutsche (cf. Scholz et al. 2015). Diese Zahlen erklären, warum die dänische Rücklaufquote höher ist. Die dänischen Hotelmanager_innen fühlen sich wie erwartet eher vom Fragebogen angesprochen als die deutschen, die verhältnismäßig wenig dänische Gäste bewirten. Insgesamt haben 267 deutsche Hoteldirektor(inn)en den Fragebogen ausgefüllt (11,1 \%).

\begin{tabular}{|c|c|c|c|}
\hline & \multicolumn{3}{|l|}{ Hotels } \\
\hline & \multicolumn{2}{|l|}{$\mathrm{D}$} & $\mathrm{DK}$ \\
\hline \multirow{3}{*}{ Bruttoansatz beim 1. Durchgang } & HH: 372 & B: 569 & \multirow{2}{*}{--} \\
\hline & NS: 724 & SH: 919 & \\
\hline & \multicolumn{2}{|c|}{ Insg.: 2584} & Insg.: 917 \\
\hline Unzustellbar & \multicolumn{2}{|l|}{177} & 45 \\
\hline \multirow{3}{*}{ Bereinigte Stichprobe } & HH: 366 & B: 559 & \multirow{2}{*}{--} \\
\hline & NS: 714 & SH: 768 & \\
\hline & \multicolumn{2}{|c|}{ Insg.: 2407} & Insg.: 872 \\
\hline Rücklauf nach 1. Durchgang & \multicolumn{2}{|l|}{168} & 130 \\
\hline Bruttoansatz beim 2. Durchgang & \multicolumn{2}{|l|}{2407} & 872 \\
\hline Unzustellbar & \multicolumn{2}{|l|}{--} & 9 \\
\hline
\end{tabular}




\begin{tabular}{|l|l|l|}
\hline Bereinigte Stichprobe & -- & 863 \\
\hline Rücklauf nach 2. Durchgang & 56 & 70 \\
\hline Rücklauf nach 3. Durchgang & 43 & -- \\
\hline $\begin{array}{l}\text { Gesamtrücklauf } \\
\text { Prozent }\end{array}$ & 267 & 200 \\
$23 \%$
\end{tabular}

Tabelle 6: Rücklauf der Fragebogenaktion unter Hotels

Von den deutschen Hoteldirektionen, die den Fragebogen ausgefüllt haben, gaben $35 \%$ an, dass ihr Hotel sich in Schleswig-Holstein befände (11\% in Hamburg, $23 \%$ in Niedersachsen und $18 \%$ in Berlin). Außergewöhnlich viele deutsche Hotelmanager_innen haben die Frage nach dem Bundesland, in dem ihr Hotel sich befindet, nicht beantwortet (13\%). Im Vergleich zur Frage nach der Frequenz der Besuche dänischer Gäste, die keine fehlenden Fälle aufweist, ist die häufige Verweigerung der Antwort auf die Bundeslandfrage sehr auffällig, zumal die anderen Fragen auch von (fast) allen Informant_innen beantwortet wurden. Noch auffälliger wirkt diese im Ländervergleich: Nur 4 \% der dänischen Hotelmanager_innen haben die Frage nach ihrer Postnummer nicht beantwortet.

Der Großteil der dänischen ausgefüllten Fragebögen kam aus der Region des südwestlichen Jütlands, aus Fünen und den umliegendem Inseln, aus Nordjütland, aus der Region des nördlichen Seelands und Bornholm sowie aus der Region des übrigen Seelands und der umliegenden Inseln.

\subsection{Auswertung und Interpretation der Umfrageergebnisse}

Die Variable Häufigkeit der Kontakte mit Hotelgästen ist im vorliegenden Beitrag ein objektiver Indikator von Fremdsprachenbedarf. Es bietet sich an, die Frage nach der Frequenz der Kontakte mit deutschen bzw. dänischen Hotelgästen als Filter einzusetzen. Nur wenn ein Informant angegeben hat, dass in seinem Hotel mindestens regelmäßig deutsche bzw. dänische Gäste übernachten, wurde sein Fragebogen in die Berechnungen einbezogen. Dreiviertel (77,5 \%) der befragten dänischen Hotels haben regelmäßig bis sehr oft deutsche Gäste. Dagegen haben nur knapp die Hälfte (44\%) der befragten deutschen Hotels regelmäßig bis sehr oft dänische Gäste.

\subsubsection{Selbsteingestufte Deutsch- bzw. Dänischkenntnisse der Hotelmanager_innen}

Von den 121 deutschen Manager_innen, die regelmäßig bis sehr oft persönlich Kontakt mit dänischen Gästen haben, geben ca. $68 \%$ an, keine Dänischkenntnisse zu haben (ca. $23 \%$ geringe und ca. $9 \%$ bessere als geringe). Die meisten dieser Manager_innen (ungefähr Zweidrittel) verfügen also nicht über Dänischkenntnisse. Von den 131 dänischen Manager_innen, die mindestens regelmäßig Kontakt mit deutschen Hotelgästen haben, haben nur $1 \%$ keine Deutschkenntnisse (18\% geringe und $81 \%$ bessere als geringe). Wie erwartet, beherrschen dänische Hoteldirektor_innen eher Deutsch als deutsche Dänisch. 


\subsubsection{Deutsch- bzw. Dänischgebrauch der Hotelmanager_innen}

Wie oft wird Dänisch von den befragten deutschen Managern_innen, die regelmäßig bis sehr häufig Kontakte mit dänischen Gästen haben, verwendet? $5 \%$ dieser Manager_innen verwenden (fast) immer Dänisch, 4 \% regelmäßig und $10 \%$ selten. Somit verwenden $81 \%$ nie Dänisch. Die Verteilungen für Deutsch und Englisch sehen folgendermaßen aus: Mehr als ein Drittel der deutschen Manager_innen gebrauchen (fast) immer Deutsch, die Hälfte regelmäßig und etwas weniger als ein Zehntel selten. Ca. $28 \%$ teilen mit, sich (fast) immer der englischen Sprache zu bedienen (ca. $60 \%$ regelmäßig, ca. $9 \%$ selten und ca. $3 \%$ nie). Zusammenfassend lässt sich sagen, dass der Dänischgebrauch (Adaptation) im norddeutschen Hotelgewerbe die Ausnahme ist. Im Kontakt mit dänischen Gästen bilden der Deutsch- und Englischgebrauch, die zahlenmäßig fast deckungsgleich sind, die Regel.

Die dänischen Vergleichszahlen: $6 \%$ der dänischen Hoteldirektor_innen mit mindestens regelmäßigen Kontakten mit deutschen Gästen verwenden nie Deutsch, $19 \%$ selten, 22 \% regelmäßig und $53 \%$ (fast) immer. $63 \%$ wählen nie Dänisch, $25 \%$ selten, $7 \%$ regelmäßig und $5 \%$ (fast) immer. Englisch (Standardisierung) wird viel häufiger als Dänisch (NichtAdaptation) gebraucht, aber am ehesten wird Deutsch gebraucht. Von den dänischen Hoteldirektor_innen im regelmäßigen bis sehr häufigen Kontakt mit deutschen Gästen verwendet mehr als die Hälfte (fast) immer Deutsch für diesen Kontakt, während nur ein Drittel (fast) immer Englisch gebraucht (34 \% (fast) immer Englisch, $27 \%$ regelmäßig, $33 \%$ selten und $6 \%$ nie).

\subsubsection{Bedarf der Hotelmanager_innen an Deutsch- bzw. Dänischkenntnissen}

Der Begriff „Dänischbedarf“ bzw. „Deutschbedarf“ (Bedarf an Dänisch- bzw. Deutschkenntnissen) wurde - wie in Kapitel 2.2 erwähnt - fünffach operationalisiert. Im Folgenden werden die fünf Arten von Dänischbedarf deutscher Manager_innen in Kurzform formuliert. 


\begin{abstract}
Dänischbedarf deutscher Hotelmanager_innen
Als Filter: objektiver Dänischbedarf deutscher Hotelmanager_innen

$=$ mitgeteilte persönliche Kontakte mit dänischen Gästen.

Subjektiver Dänischbedarf deutscher Hotelmanager_innen mit regelmäßigen bis sehr häufigen Kontakten zu dänischen Gästen.

$=$ Mitteilung, dass man persönlich einen Bedarf an Dänischkenntnissen hat. Dieser Bedarf kann als ge-

deckt (man habe persönlich genug Dänischkenntnisse) oder ungedeckt (man habe keine oder zu wenig Dänischkenntnisse) betrachtet werden.
\end{abstract}

Unbewusster Dänischbedarf deutscher Hotelmanager_innen mit regelmäßigen bis sehr häufigen Kontakten zu dänischen Gästen

= Mitteilung, dass man persönlich im Hotel nie/selten Bedarf an Dänischkenntnissen hat.

Objektiver Dänischmehrbedarf deutscher Hotelmanager_innen mit regelmäßigen bis sehr häufigen

Kontakten zu dänischen Gästen

= Mitteilung, dass man persönlich nie/selten Dänisch im Kontakt mit dänischen Gästen verwendet.

Subjektiver Dänischmehrbedarf deutscher Hotelmanager_innen mit regelmäßigen bis sehr häufigen

Kontakten zu dänischen Gästen und einem subjektiven Dänischbedarf

= Mitteilung, dass man persönlich keine/eher zuwenig Dänischkenntnisse hat.

39 befragte deutsche Hotelmanager_innen haben einen sehr häufigen objektiven Dänischbedarf und 82 einen regelmäßigen. Ich gehe - wie bereits erwähnt - davon aus, dass die Personen, die die Muttersprache seines Gegenübers beherrschen, am besten für die geschäftliche Kommunikation mit ihm ausgestattet sind, weil sie die Adaptationsstrategie anwenden können. Sehen deutsche Hoteldirektor_innen mit mindestens regelmäßigen Kontakten mit dänischen Gästen einen Bedarf an Kenntnissen der Muttersprache dieser Gäste (subjektiver Dänischbedarf)? $40 \%$ geben an, keinen Bedarf an Dänischkenntnissen zu haben. Sie sind sich ihres Dänischbedarfs nicht bewusst. 60 \% sehen einen Bedarf an Dänischkenntnissen. Ungefähr die Hälfte der Manager_innen mit einem subjektiven Dänischbedarf meint zu wenig oder keine Dänischkenntnisse zu haben und hat somit einen subjektiven Mehrbedarf an Dänischkenntnissen, während $9 \%$ der Meinung sind, genug Dänischkenntnisse zu haben. Informant_innen, die mitteilen, dass sie nie/nur selten Dänisch benutzen, haben einen objektiven Dänischmehrbedarf. Ca. $92 \%$ der befragten deutschen Hotelmanager_innen, die regelmäßig bis sehr oft mit dänischen Gästen kommunizieren, haben einen solchen Bedarf.

Fazit: $60 \%$ der befragten Manager_innen mit regelmäßigen bis sehr häufigen Kontakten mit Dänen im Hotel sind sich eines eigenen Dänischbedarfs bewusst (subjektiver Bedarf). Das Ergebnis, dass deutlich mehr als die Hälfte der Manager_innen mit Kontakten zu dänischen Gästen für sich selbst einen Dänischbedarf sieht, ist als positiv zu betrachten. Dass ungefähr die Hälfte der befragten Manager_innen mit regelmäßigen bis sehr häufigen Kontakten zu dänischen Hotelgästen einen subjektiven Mehrbedarf an Dänischkenntnissen hat, ist genauso positiv: ca. 50 \% hätten gern (mehr) Dänischkenntnisse. Sie müssen nicht für den Erwerb des Dänischen sensibilisiert werden. Dies sind insgesamt 60 Personen. 33 davon haben keine, 22 geringe und 5 bessere als geringe Dänischkenntnisse.

Die befragten dänischen Hoteldirektor_innen kommen viel häufiger in Berührung mit Gästen aus Deutschland als ihre Kollegen in Deutschland mit Gästen aus Dänemark. 95 \% der däni- 
schen Hotelmanager_innen mit Kontakten zu deutschen Gästen haben einen subjektiven Bedarf und sind sich somit ihres Deutschbedarfs bewusst, was als sehr positiv gesehen werden muss. Nur $5 \%$ behaupten also im Hotel keinen Bedarf an Deutschkenntnissen zu haben. Diese zeigen einen unbewussten Bedarf. $22 \%$ wünschen sich (mehr) Deutschkenntnisse. Dies sind insgesamt 28 Personen. 2 davon haben keine Deutschkenntnisse, 14 geringe und 12 bessere als geringe.

Der objektive Mehrbedarf dänischer Hoteliers liegt bei $32 \%$ (die norddeutsche Vergleichszahl: 92 \%). In Dänemark ist die Zahl der Hoteliers, die regelmäßig bis sehr oft mit deutschen Gästen in Berührung kommt und im Umgang mit ihnen selten oder nie Deutsch verwendet, verhältnismäßig gering. Wie erwartet, spielt die deutsche Sprache im dänischen Hotelgewerbe eine viel größere Rolle als die dänische Sprache im deutschen. Die dänischen Hoteliers, die gemessen an ihren Kontakten mit Gästen aus dem Nachbarland - einen Mehrbedarf an Deutschkenntnissen haben, sind sich ihres Bedarfs auch bewusst, während die deutschen in der gleichen Situation sich ihres Bedarfs an Dänischkenntnissen viel weniger bewusst sind.

\subsubsection{Wissen der Hotelmanager_innen um die deutsche bzw. dänische Mentalität}

Ca. $6 \%$ der befragten deutschen Hotelmanager_innen mit regelmäßigen bis häufigen Kontakten zu dänischen Gästen teilen mit, nicht über Wissen um die dänische Mentalität zu verfügen. Ca. $6 \%$ meinen sehr viel und $16 \%$ eher viel über die dänische Mentalität zu wissen. Die restlichen positionieren sich dazwischen (27\% teils wenig/teils viel, $22 \%$ eher wenig, $23 \%$ sehr wenig und $6 \%$ kein Wissen).

Das Wissen in Dänemark über Deutschland scheint viel umfangreicher zu sein als das Wissen in Norddeutschland über Dänemark. Nur $2 \%$ der befragten dänischen Hotelmanager_innen mit regelmäßigen bis häufigen Kontakten zu deutschen Gästen geben an, sehr wenig Wissen um die deutsche Mentalität zu haben. $11 \%$ meinen über eher wenig Kenntnisse der deutschen Mentalität zu verfügen. Teils wenig/teils viel kreuzten $24 \%$ als Antwort an, $38 \%$ eher viel und $24 \%$ sehr viel.

\subsubsection{Bedarf der Hotelmanager_innen an Wissen um die deutsche bzw. dänische Men- talität}

Ca. $85 \%$ der deutschen Hotelmanager_innen mit regelmäßigem bis sehr häufigen Kontakten zu dänischen Gästen haben einen subjektiven Bedarf an Wissen um die dänische Kultur: Sie teilen mit, einen persönlichen Bedarf an Wissen um die dänische Mentalität zu haben. Der subjektive Bedarf norddeutschen Hotelmanager_innen an Wissen um die dänische Mentalität ist also sehr groß. Ca. 56 \% der Informant_innen behaupten, über kein/zu wenig Wissen um die dänische Mentalität zu verfügen (subjektiver Dänischmehrbedarf). Insgesamt sind dies 68 Personen. 2 davon meinen, bereits sehr viel Wissen um die dänische Mentalität zu haben und 4 eher viel. Nur 3 der 68 gehen davon aus, kein Wissen um die dänische Mentalität zu haben. Die anderen (59) lassen sich dazwischen positionieren. Sie haben „sehr wenig“, „eher wenig“ oder „teils wenig/teils viel“ als Antwort angekreuzt. $15 \%$ zeigen einen unbewussten Bedarf.

Interessant ist der Vergleich mit dem Bedarf an Dänischkenntnissen. Ca. 85 \% der norddeutsche Hotelmanager_innen haben einen persönlichen subjektiven Bedarf an Wissen um die 
dänische Mentalität und ca. $60 \%$ haben einen Bedarf an Dänischkenntnissen. Der subjektive Kulturbedarf ist also viel größer als der subjektive Sprachbedarf.

Es müssen noch die Antworten der deutschen mit denen der dänischen Befragten verglichen werden. Bei ungefähr gleichem subjektivem Bedarf an Wissen um die Mentalität im Nachbarland ( $85 \%$ versus $95 \%$ ) ist der deutsche subjektive Mehrbedarf an Wissen um die dänische Mentalität fast viermal höher als der dänische subjektive Mehrbedarf an Wissen um die deutsche Mentalität (15\%). Die dänischen Informant_innen gehen eher als die deutschen davon aus, genügend Wissen um das Nachbarland zu haben. Der deutsche unbewusste Bedarf ist auch dreimal höher als der dänische (5\%). Der Mehrbedarf an Wissen um die dänische Mentalität ist im norddeutschen Hotelgewerbe sehr hoch. Wenn subjektiver Mehrbedarf und unbewusster Bedarf addiert werden, ergibt dies ca. 70 \% (die Vergleichszahl für Dänemark: $20 \%)$.

\subsubsection{Deutsch- bzw. Dänischkenntnisse des Hotelpersonals mit Gästekontakt}

Bei 19 \% liegt der Anteil der deutschen Hotels, die regelmäßig bis sehr oft dänische Gäste empfangen und eine Mitarbeiter_in mit besseren als geringen Dänischkenntnissen haben. Hinzu kommen die Hotels, die zwei oder drei Mitarbeiter_innen mit besseren als geringen Dänischkenntnissen haben ( $8 \%$ bzw. $3 \%$ von insgesamt 118 Hotels mit mindestens regelmäBigen Besuchen dänischer Gäste). Also: $30 \%$ haben mindestens eine solche Mitarbeiter_in. Der Anteil der deutschen Hotels mit mindestens einer Mitarbeiter_in mit nur geringen Dänischkenntnissen ist höher: $40 \%$. $17 \%$ haben eine Mitarbeiter_in, die dänischsprachige EMails von Gästen auf Dänisch beantworten kann, während $8 \%$ zwei oder drei solcher Mitarbeiter_innen haben. Ca. $68 \%$ teilen mit, keine Mitarbeiter_innen mit besseren als geringen Dänischkenntnissen zu haben. Ca. $74 \%$ geben an, dass keine/keiner ihrer Mitarbeiter_innen dänischsprachige E-Mails von Gästen auf Deutsch beantworten kann.

Auch die Deutschkenntnisse des Personals der dänischen Hotels mit regelmäßigen bis sehr häufigen Kontakten zu deutschen Gästen wurden unter die Lupe genommen (insgesamt 133). $14 \%$ dieser Hotels haben eine Mitarbeiter_in mit besseren als geringen Deutschkenntnissen, $20 \%$ mit zwei und $23 \%$ mit drei. $57 \%$ der dänischen Hotels haben somit mindestens eine Mitarbeiter_in mit Kontakt zu deutschen Gästen, die bessere als geringe Deutschkenntnisse hat. Der Anteil der dänischen Hotels mit mindestens einer Mitarbeiter_in mit nur geringen Deutschkenntnissen ist etwas niedriger: $51 \%$. In drei von vier dänischen Hotels arbeitet jemand, der die deutschsprachigen E-Mails von Gästen auf Deutsch beantworten kann. Nur jedes vierte deutsche Hotel hat eine Angestellte, die eine dänischsprachige Antwort auf EMails verfassen kann. Es ist unverkennbar, dass Deutsch eine viel größere Rolle in dänischen Hotels spielt als die dänische Sprache in deutschen. Das Deutsche bewirkt eher Adaptation als das Dänische.

\subsubsection{Deutsch- bzw. Dänischgebrauch des Hotelpersonals mit Gästekontakt}

Im Folgenden werden genaue Angaben zur Häufigkeit der Verwendung des Deutschen, Dänischen und Englischen durch die befragten deutschen und dänischen Hotels gemacht. Es fließen jeweils nur die Daten der Hotels mit regelmäßigen Besuchen von Gästen aus dem Nachbarland in die Analyse ein. 
Von den 118 betreffenden deutschen Hotels verwenden im Kontakt mit dänischen Gästen

19 \% selten Dänisch

$8 \%$ regelmäßig Dänisch

$3 \%$ (fast) immer Dänisch

$6 \%$ selten Deutsch

$54 \%$ regelmäßig Deutsch

$33 \%$ (fast) immer Deutsch

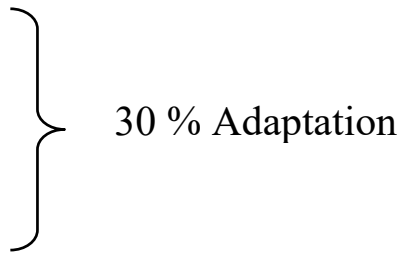

$6 \%$ selten Englisch

69 \% regelmäßig Englisch

$21 \%$ (fast) immer Englisch

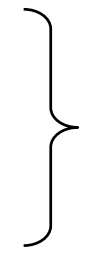

$93 \%$ Nicht-Adaptation

Insgesamt verwenden $11 \%$ regelmäßig bis (fast) immer Dänisch, $87 \%$ regelmäßig bis (fast) immer Deutsch und 90 \% regelmäßig bis (fast) immer Englisch.

Von den 133 dänischen Hotels, die regelmäßig bis sehr oft deutsche Gäste haben, verwenden im Kontakt mit deutschen Gästen

$23 \%$ selten Deutsch

$45 \%$ regelmäßig Deutsch

$27 \%$ (fast) immer Deutsch

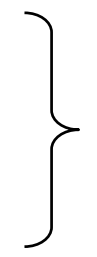

$95 \%$ Adaptation

29 \% selten Dänisch

$13 \%$ regelmäßig Dänisch

$10 \%$ (fast) immer Dänisch

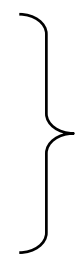

$52 \%$ Nicht-Adaptation

$23 \%$ selten Englisch

$40 \%$ regelmäßig Englisch

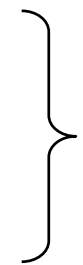

$98 \%$ Englisch als Lingua franca

$35 \%$ (fast) immer Englisch

Fazit: $72 \%$ der dänischen Hotels verwenden regelmäßig bis (fast) immer Deutsch im Kontakt mit deutschen Gästen, 23 \% regelmäßig bis (fast) immer Dänisch und $75 \%$ regelmäßig bis (fast) immer Englisch. Adaptation und Standardisierung sind somit die beiden Sprachwahlstrategien, die von der Mehrheit des dänischen Hotelpersonals im Umgang mit deutschen Gästen gleich häufig verfolgt werden. Das deutsche Hotelpersonal bevorzugt fast ohne Ausnahme die Nicht-Adaptation und Standardisierung. 


\subsubsection{Bedarf des Hotelpersonals mit Gästekontakt an Deutsch- bzw. Dänischkenntnis- sen}

Diesmal werden einleitend die fünf gemessenen Arten von Dänischbedarf in Kurzform aufgelistet.

Objektiver Dänischbedarf als Filter
= mitgeteilte Besuche dänischer Gäste

Objektiver Dänischmehrbedarf deutscher Hotels mit regelmäßigen bis häufigen Besuchen dänischer Gäste

= Mitteilung, dass nie/selten Dänisch im Kontakt mit dänischen Gästen verwendet wird

Subjektiver Dänischbedarf deutscher Hotels mit regelmäßigen bis häufigen Besuchen dänischer Gäste

$=$ Mitteilung, dass das Hotel einen Bedarf an Dänischkenntnissen hat. Dieser Bedarf kann als gedeckt (das Hotelpersonal hat genügend Dänischkenntnisse) oder ungedeckt betrachtet werden (das Personal hat keine oder zuwenig Dänischkenntnisse)

Subjektiver Dänischmehrbedarf deutscher Hotels mit regelmäßigen bis häufigen Besuchen dänischer Gäste und subjektivem Dänischbedarf

$=$ Mitteilung, dass im Hotel keine/eher zu wenig Dänischkenntnisse vorhanden sind

Unbewusster Dänischbedarf deutscher Hotels mit regelmäßigen bis häufigen Besuchen dänischer Gäste

$=$ Mitteilung, dass das Hotel nie/selten Bedarf an Dänischkenntnissen hat

Schauen wir uns zunächst den subjektiven Dänischbedarf an. Ein Drittel der 118 deutschen Hotelmanager_innen, deren Hotels regelmäßig bis sehr oft von Dänen besucht werden, meint, ihr Hotel brauche die nächsten Jahre keine Dänischkenntnisse. Ca. $42 \%$ setzen einen seltenen Bedarf, ca. $20 \%$ einen regelmäßigen und ca. $6 \%$ einen sehr häufigen voraus. Somit haben $26 \%$ laut eigener Aussagen einen mindestens regelmäßigen Dänischbedarf.

Die Vergleichszahlen für Dänemark: $2 \%$ der Informant_innen sehen keinen Deutschbedarf, $21 \%$ einen seltenen, $54 \%$ einen regelmäßigen und $23 \%$ einen sehr häufigen. Die dänischen Hoteliers sind sich in viel größerem Maße als die norddeutschen dessen bewusst, dass ihr Personal Kenntnisse der Sprache des Nachbarlandes benötigt.

$51 \%$ der deutschen Hotelmanager_innen sehen für ihr Hotel mit regelmäßigen bis sehr häufigen Besuchen dänischer Gäste zum Zeitpunkt der Befragung einen Bedarf an Dänischkenntnissen. Davon behaupten ca. $37 \%$ mindestens eine(n) Mitarbeiter_in mit besseren als geringen Dänischkenntnissen und $42 \%$ mindestens eine Mitarbeiter_in mit geringen Dänischkenntnissen zu haben. Dies deutet auf einen subjektiven Mehrbedarf hin. Die Auswertung zeigt tatsächlich, dass $43 \%$ der Hotels, die regelmäßig bis sehr häufig von Dänen frequentiert werden, einen subjektiven Dänischmehrbedarf haben. Sie geben an, dass ihr Personal über keine oder zu wenig Dänischkenntnisse verfügt. $8 \%$ gehen davon aus, dass ihr Dänischbedarf gedeckt ist.

90 \% der deutschen Hotels, die regelmäßig bis sehr häufig dänische Gäste zu Besuch haben, zeigen einen objektiven Dänischmehrbedarf, d. h. sie verwenden nie/selten Dänisch. 49 \% haben einen unbewussten Dänischbedarf. 
90 \% der 133 dänischen Befragten, deren Hotels mindestens regelmäßig deutsche Gäste empfangen, geben an, einen Bedarf an Deutschkenntnissen zu haben. 10\% haben somit einen unbewussten Bedarf. Einen subjektiven Mehrbedarf haben $43 \%$. $47 \%$ gehen davon aus, dass ihr Deutschbedarf gedeckt ist, indem sie angeben, über genügend Personal mit Deutschkenntnissen zu verfügen. Ein Drittel der dänischen Hotels hat einen objektiven Deutschmehrbedarf, d. h. sie verwenden selten oder nie Deutsch, obwohl sie regelmäßig bis sehr häufig von Gästen aus Deutschland frequentiert werden.

Der dänische subjektive Deutschbedarf ist mit $90 \%$ fast doppelt so groß wie der deutsche subjektive Dänischbedarf, während es beim subjektiven Mehrbedarf an Kenntnissen der Sprache des Nachbarlandes keinen zahlenmäßigen Unterschied gibt (43\%). Als gedeckt betrachten $47 \%$ der dänischen Hoteliers den Deutschbedarf in ihren Hotels. Hingegen halten nur $8 \%$ ihrer norddeutschen Kollegen den Dänischbedarf ihrer Hotels für gedeckt. Einen Dänischbedarf sieht ungefähr die Hälfte der deutschen Befragten, deren Hotels mindestens regelmäßig von dänischen Gästen besucht werden. Wenn man diese Frequenz der Besuche dänischer Hotelgäste in Norddeutschland als Indikator für Bedarf betrachtet, hat die andere Hälfte der Befragten einen unbewussten Dänischbedarf. Nur $10 \%$ der dänischen Hoteliers haben dagegen einen unbewussten Deutschbedarf. Das dänische Hotelgewerbe muss also nicht für den Erwerb des Deutschen als Fremdsprache sensibilisiert werden. In Norddeutschland müsste schon noch Sensibilisierungsarbeit geleistet werden, damit mehr Hotelangestellte für ihren Beruf Dänisch lernen (können).

\subsubsection{Wissen des Hotelpersonals mit Gästekontakt um die deutsche bzw. dänische Mentalität}

Gleich viele deutsche Hoteliers, in deren Hotels Dänen übernachten, meinen, sie hätten Personal mit Wissen (47 \%) bzw. sie hätten Personal ohne Wissen (45 \%) um die dänische Mentalität. $8 \%$ geben an, sie wüssten nicht, ob ein solches Wissen in ihren Hotels vorhanden sei.

Die Zahlen für die dänische Seite sehen völlig anders aus. Auf die Frage „Hat Ihr Personal mit Gästekontakt Ihrer Meinung nach Wissen über die deutsche Mentalität?“` antworten $71 \%$ „eher ja“ und $16 \%$,eher nein“ und $13 \%$,weiß ich nicht“.

\subsubsection{Bedarf des Hotelpersonals mit Gästekontakt an Wissen über die deutsche bzw. dänische Mentalität}

Fangen wir mit dem subjektiven Bedarf des Hotelpersonals an Fremdkulturwissen an, der sich an Tabelle 7 ablesen lässt.

\begin{tabular}{|l|l|l|}
\hline $\begin{array}{l}\text { Wie viel Wissen über die } \\
\text { dänische bzw. deutsche Men- } \\
\text { talität benötigt Ihr Hotel? }\end{array}$ & in Norddeutschland & in Dänemark \\
\hline kein Wissen & $5 \%$ & $2 \%$ \\
\hline sehr wenig & $11 \%$ & $8 \%$ \\
\hline eher wenig & $38 \%$ & $27 \%$ \\
\hline teils wenig / teils viel & $25 \%$ & $42 \%$ \\
\hline
\end{tabular}

ISSN 1615-3014 


\begin{tabular}{|l|l|l|}
\hline eher viel & $18 \%$ & $19 \%$ \\
\hline sehr viel & $3 \%$ & $3 \%$ \\
\hline & $100 \%$ & $100 \%$ \\
\hline
\end{tabular}

Tabelle 7: Subjektiver Bedarf an Kulturwissen des Hotelpersonals

Die befragten deutschen Hotelmanager_innen geben zu $5 \%$ an, dass ihr Hotel mit mindestens regelmäßigen Aufenthalten von Dänen kein Wissen über die dänische Mentalität für den Kontakt mit dänischen Gästen benötigt. $49 \%$ kreuzten an, dass ihr Hotel sehr wenig oder eher wenig Wissen benötigt. $21 \%$ sind der Meinung, dass ihr Hotel eher viel oder sehr viel solchen Wissens über die dänische Mentalität für den Umgang mit dänischen Gästen braucht.

Dänische Hotels, die regelmäßig oder sehr oft deutsche Gäste haben, geben zu $2 \%$ an, kein Wissen über die deutsche Mentalität im Umgang mit deutschen Gästen zu brauchen. Weitere $34 \%$ kreuzten an, nur sehr wenig oder eher wenig Wissen um die deutsche Mentalität zu benötigen. $22 \%$ der dänischen Hotelmanager_innen gaben an, eher viel und sehr viel Wissen über die deutsche Mentalität zu brauchen. Die Unterschiede zwischen den Antworten der deutschen und dänischen Informanten sind nicht sehr groß. Die Dänen sehen einen etwas gröBeren Bedarf an Wissen um die Mentalität im Nachbarland.

Eine zweite Frage nach dem subjektiven Bedarf konnte nur mit ,ja“ oder ,nein“ beantwortet werden. $19 \%$ der deutschen Hotels mit mindestens regelmäßigen Aufenthalten von Dänen behaupten, das Personal hat keinen Bedarf an Wissen über die deutsche Mentalität (diese haben einen unbewussten Bedarf). $81 \%$ behaupten also einen Bedarf an Wissen um die dänische Mentalität zu haben. $51 \%$ davon haben einen subjektiven Mehrbedarf. Sie geben an, in ihren Hotels sei kein/zu wenig Wissen um die dänische Mentalität vorhanden. $30 \%$ haben die Antwort „das Personal hat genug Wissen um die dänische Mentalität“، angekreuzt.

$7 \%$ der befragten dänischen Hotels mit regelmäßigen/sehr häufigen Besuchen deutscher Gäste haben einen unbewussten Bedarf (sie teilen mit, keinen Bedarf an Wissen über die dänische Mentalität zu haben). Somit haben $93 \%$ einen subjektiven Bedarf. $28 \%$ haben einen Mehrbedarf, während $65 \%$ meinen, genug Kenntnis der deutschen Kultur zu haben.

Der subjektive Mehrbedarf an Wissen um die Kultur des Nachbarlandes ist im norddeutschen Hotelgewerbe größer als der gleiche Bedarf im dänischen. Der subjektive Bedarf, den dänische Hoteliers sehen, ist größer. Der dänische unbewusste Bedarf ist daher kleiner. Diese Ergebnisse deuten darauf hin, dass das norddeutsche Gewerbe einen verhältnismäßig großen ungedeckten Bedarf an Wissen um die dänische Kultur hat.

\section{$5 \quad$ Fragebogenaktion unter Firmen in Schleswig-Holstein und Hamburg}

\subsection{Fragebogenvariable, Stichprobe, Rücklauf und fehlende Fälle}

Die Fragen, welche die Firmenmanager_innen beantworteten, waren mutatis mutandis die Fragen des Fragebogens für Hotelmanager_innen. Die Variablen können - so wie sie für die Fragebogenaktion unter Hotelmanager_innen formuliert wurden - bis auf die Wörter „Hotelgäste“ und „Hotelpersonal“ eins zu eins übernommen werden. „Kontakte mit Hotelgästen“ muss ersetzt werden durch „Kontakte (Export, Import, Beratung, Kooperation, etc.) mit Per- 
sonen in Dänemark bzw. Deutschland“ und „Hotelpersonal mit Gästekontakt“ durch „Mitarbeiter_innen, die bei den Geschäften mit ausländischen Unternehmen mitwirken““.

Im ersten Durchgang wurden 1257 Firmen aus Schleswig-Holstein und Hamburg per Brief erreicht (bereinigte Stichprobe). 221 davon haben den Fragebogen ausgefüllt zurückgeschickt. In Deutschland bekam die bereinigte Stichprobe der Firmen ein Erinnerungsschreiben, was die Zahl der ausgefüllten Fragebögen um 13,5 \% steigerte (Gesamtrücklaufquote $28 \%$ ). Von den 419 erreichten dänischen Unternehmen füllten nach nur einem Durchgang 130 den Fragebogen aus (Rücklaufquote $32 \%$ ). Wegen dieser hohen Rücklaufquote wurde hier auf einen zweiten Durchgang verzichtet.

\begin{tabular}{|l|l|l|}
\hline \multicolumn{2}{|l|}{ Firmen } & DK \\
\hline & D & 461 \\
\hline Bruttoansatz beim 1. Durchgang & 1270 & 42 \\
\hline Unzustellbar & 13 & 419 \\
\hline Bereinigte Stichprobe & 1257 & 130 \\
\hline Rücklauf nach 1. Durchgang & 221 & -- \\
\hline Bruttoansatz beim 2. Durchgang & 1257 & -- \\
\hline Unzustellbar & 1 & -- \\
\hline Bereinigte Stichprobe & 1256 & -- \\
\hline Rücklauf nach 2. Durchgang & 127 & 136 \\
\hline Gesamtrücklauf & 348 & $32 \%$ \\
\hline Prozent & $28 \%$ & \\
\hline
\end{tabular}

Tabelle 8: Rücklauf der Fragebogenaktion unter Firmen

Von den befragten deutschen Manager_innen gab ca. $50 \%$ an, dass ihr Unternehmen sich in Schleswig-Holstein befinde, die andere Hälfte sind Hamburger Unternehmen. Anders als die Hotelmanager_innen haben nur 10 der 348 befragten deutschen Firmenmanager_innen die Frage nach dem Bundesland nicht beantwortet. Die anderen Fragen weisen noch weniger fehlende Fälle auf.

\subsection{Auswertung und Interpretation der Umfrageergebnisse}

Wie erwähnt ist die Variable Häufigkeit der Kontakte im vorliegenden Beitrag ein objektiver Indikator von Fremdsprachenbedarf und wird als Filter benutzt. Ca. $44 \%$ der (befragten) deutschen Firmen treten regelmäßig bis sehr häufig in Kontakt mit Dänen, während fast $90 \%$ (also doppelt so viele) der dänischen Unternehmen in Berührung mit Deutschen kommen. 


\subsubsection{Selbsteingestufte Deutsch- bzw. Dänischkenntnisse der Firmenmanager_innen}

Der Anteil der 157 deutschen Manager_innen mit mindestens regelmäßigem persönlichem Kontakt zu Dänen, die nach eigener Aussage keine Dänischkenntnisse haben, beträgt ca. $65 \%$. Ein Fünftel verfügt über geringe Dänischkenntnisse, ein Zehntel über bessere als geringe. $83 \%$ der 110 befragten dänischen Manager_innen, die mindestens regelmäßig geschäftlich mit Deutschen kommunizieren, haben bessere als geringe Deutschkenntnisse, $17 \%$ geringe und nur $7 \%$ keine. Überraschend ist es nicht, dass dänische Manager_innen verhältnismäßig gute Deutschkenntnisse haben, während deutsche mehrheitlich nicht über Dänischkenntnisse verfügen.

\subsubsection{Deutsch- bzw. Dänischgebrauch der Firmenmanager_innen}

Das präsentierte Datenmaterial bezieht sich auch hier auf die Manager_innen, die mindestens regelmäßig geschäftlich mit Dänen bzw. Deutschen zu tun haben. Wie oft verwenden die erfassten Deutschen Dänisch im Kontakt mit Dänen? $79 \%$ nie, $8 \%$ selten, $7 \%$ regelmäßig und $6 \%$ (fast) immer. Es ist unverkennbar, dass Dänisch von Manager_innen aus SchleswigHolstein und Hamburg, die geschäftliche Kontakte zu Deutschland unterhalten, kaum verwendet wird. Bezüglich des Deutschgebrauchs (Nicht-Adaptationen) kann gesagt werden, dass ein Fünftel nie Deutsch verwendet, ein Viertel selten, etwas mehr als ein Drittel regelmäßig und etwas weniger als ein Fünftel (fast) immer. Englisch wird ein wenig häufiger als Deutsch gebraucht, von ca. $16 \%$ nie, von $20 \%$ selten, von $32 \%$ regelmäßig und auch von $32 \%$ (fast) immer.

Verglichen mit den deutschen Befragten ist die Zahl der dänischen Manager_innen, die (fast) immer die Adaptationsstrategie einsetzen um 37\% höher: $43 \%$ verwenden (fast) immer Deutsch, $22 \%$ regelmäßig, $13 \%$ selten und $22 \%$ nie. Während vier Fünftel der Deutschen mindestens gelegentlich die Nicht-Adaptationsstrategie wählen, wird die Nicht-Adaptation von den meisten Dänen nie praktiziert: $88 \%$ verwenden nie Dänisch, $9 \%$ selten, 1,5 \% regelmäßig und auch 1,5\% (fast) immer. Englisch verwenden sie weniger häufig als Deutsch. Die Verteilung für die Verwendung des Englischen als Lingua franca (Standardisierung) sieht folgendermaßen aus: $23 \%$ (fast) immer, $25 \%$ regelmäßig, $21 \%$ selten und $30 \%$ nie.

\subsubsection{Bedarf der Firmenmanager_innen an Deutsch bzw. Deutschkenntnissen}

Es werden deutlichkeitshalber kurz die fünf Arten von Deutschbedarf am Beispiel der dänischen Manager_innen aufgelistet. 
Deutschbedarf dänischer Firmenmanager_innen

Als Filter: objektiver Deutschbedarf dänischer Firmenmanager_innen

$=$ mitgeteilte persönliche Kontakte mit deutschen Geschäftspartner_innen.

Subjektiver Deutschbedarf dänischer Firmenmanager_innen mit regelmäßigen bis sehr häufigen

Kontakten zu deutschen Geschäftspartner_innen

$=$ Mitteilung, dass man persönlich einen Bedarf an Deutschkenntnissen hat. Dieser Bedarf kann als gedeckt (man habe persönlich genug Deutschkenntnisse) oder ungedeckt (man habe keine oder zu wenig Deutschkenntnisse) betrachtet werden.

Unbewusster Deutschbedarf dänischer Firmenmanager_innen mit regelmäßigen bis sehr häufigen

Kontakten zu deutschen Geschäftspartner_innen

$=$ Mitteilung, dass man persönlich in der Firma nie/selten Bedarf an Deutschkenntnissen hat.

Objektiver Deutschmehrbedarf dänischer Firmenmanager_innen mit regelmäßigen bis sehr häu-

figen Kontakten zu deutschen Geschäftspartner_innen

$=$ Mitteilung, dass man persönlich nie/selten Deutsch im Kontakt mit deutschen Geschäfts-

partner_innen verwendet.

Subjektiver Deutschmehrbedarf dänischer Firmenmanager_innen mit regelmäßigen bis sehr häufigen Kontakten zu deutschen Geschäftspartner_innen und einem subjektiven Deutschbedarf

= Mitteilung, dass man persönlich keine/eher zuwenig Deutschkenntnisse hat.

157 der 348 befragten deutschen Manager_innen haben einen mindestens regelmäßigen objektiven Dänischbedarf. 46 dieser 157 (29\%) haben einen sehr häufigen objektiven Dänischbedarf. Ihre 136 dänischen Counterparts haben fast alle einen mindestens regelmäßigen objektiven Deutschbedarf. Von den 110 befragten dänischen Managern mit einem mindestens regelmäßigen objektiven Deutschbedarf haben 60 (55\%) einen sehr häufigen objektiven Deutschbedarf.

Nur $4 \%$ der dänischen Manager_innen mit einem mindestens regelmäßigen objektiven Deutschbedarf behaupten, im Unternehmen keinen Bedarf an Deutschkenntnissen zu haben. Somit haben $96 \%$ einen subjektiven Bedarf oder anders formuliert: ein unbewusster Deutschbedarf kommt in der dänischen Teilpopulation äußerst selten vor. $38 \%$ geben an, zu wenig oder keine Deutschkenntnisse zu haben, obwohl sie sie benötigen (=subjektiver Mehrbedarf). Bezüglich dieser 48 Personen kann gesagt werden, dass eine davon keine Deutschkenntnisse hat, während 16 geringe und 31 bessere als geringe Deutschkenntnisse haben. Es fällt auf, dass die dänischen Manager_innen sehr hohe Ansprüche an ihre Deutschkenntnisse stellen. Deutlich mehr als die Hälfte der Manager_innen, die einen Deutschmehrbedarf sehen, haben schon bessere als geringe Deutschkenntnisse. Der objektive Deutschmehrbedarf liegt bei $37 \%$.

$41 \%$ der befragten deutschen Manager_innen, die persönlich geschäftlichen Kontakt mit Dänemark haben, sind der Meinung, dass sie im Unternehmen keinen Bedarf an Dänischkenntnissen haben (=unbewusster Dänischbedarf). Diese müssten für den Erwerb von Dänischkenntnissen sensibilisiert werden, wenn man davon ausgeht, dass Adaptation die beste Strategie ist. Einen subjektiven Bedarf haben 59 \%. Diese würden keine Sensibilisierung benötigen. 62 Manager_innen (ca. $40 \%$ von 157 mit einem objektiven Bedarf) haben einen subjektiven Mehrbedarf. Davon haben 34 keine, 24 geringe und nur 4 bessere als geringe Dänischkennt- 
nisse. Diese Zahlen zeigen, dass sich deutsche Manager_innen schneller als die dänischen, was die Kenntnisse der Sprache des Nachbarlandes betrifft, zufriedengeben. Einen objektiven Dänischmehrbedarf haben $88 \%$ der Manager_innen mit einem mindestens regelmäßigen objektiven Bedarf.

\subsubsection{Wissen der Firmenmanager_innen um die deutsche bzw. dänische Mentalität}

Nach eigener Aussage haben die dänischen Manager_innen mehr Wissen um die deutsche Mentalität als ihre deutschen Kollegen um die dänische Mentalität (Filter: regelmäßige bis sehr häufige persönliche geschäftliche Kontakte mit dem Nachbarland). $18 \%$ der deutschen Manager_innen die in Kontakt mit der dänischen Kultur treten, haben eher viel Wissen um die dänische Mentalität (5\% kein Wissen, $15 \%$ sehr wenig, $29 \%$ eher wenig, $24 \%$ teils wenig/teils viel, sehr viel $9 \%$ ), während $48 \%$ der dänischen Manager_innen über eher viel Wissen um die deutsche Mentalität, mit der sie regelmäßig bis sehr häufig konfrontiert werden, verfügen ( $2 \%$ sehr wenig, $9 \%$ eher wenig, $24 \%$ teils wenig/teils viel, $17 \%$ sehr viel).

\subsubsection{Bedarf der Firmenmanager_innen an Wissen um die deutsche bzw. dänische Mentalität}

Ca. $81 \%$ der Befragten der Teilpopulation der deutschen Manager_innen, die persönlich mindestens regelmäßig geschäftliche Kontakte mit Dänemark pflegen, sehen einen Bedarf an Wissen um die dänische Mentalität (die Vergleichszahl für ihre dänische Kollegen, was deren subjektiven Bedarf an Wissen um die deutsche Mentalität betrifft: 95 \%). Wenn Kulturwissen das Bedarfsobjekt darstellt, verringert sich der Unterschied zwischen den Angaben der deutschen und dänischen Teilpopulation: Geben $59 \%$ der deutschen Befragten an, dass sie Dänischkenntnise benötigen, so sind es $81 \%$, die einen Bedarf an Wissen um die dänische Mentalität sehen. Einen Mehrbedarf an Kulturwissen haben $36 \%$ der Deutschen (Vergleichszahl für Dänemark: $27 \%$ ). Einen unbewussten Bedarf haben ca. $20 \%$ der deutschen Befragten und ca. $5 \%$ der dänischen Befragten.

\subsubsection{Deutsch- bzw. Dänischkenntnisse der Firmenangestellten}

Es wurden Firmen in Schleswig-Holstein und Hamburg sowie in Dänemark befragt. Der Anteil der deutschen Firmen, die mindestens eine Firmenangestellte_n haben, die bei Geschäften mit dänischen Firmen mitwirkt und bessere als geringe Dänischkenntnisse hat, liegt bei $24 \%$. Eine solche Mitarbeiter_in haben $12 \%$ der deutschen Firmen, zwei solcher Mitarbeiter_innen $6 \%$, drei $4 \%$, mehr als drei $2 \%$ und keine $68 \%$. Die Häufigkeitsverteilung für die schleswig-holsteinischen Firmen ist nahezu identisch mit der Häufigkeitsverteilung für die Hamburger Firmen. Dies trifft auch zu, wenn die Mitarbeiter_innen mit nur geringen Dänischkenntnissen und die Mitarbeiter_innen, die dänischsprachige E-Mails auf Dänisch beantworten können, gezählt werden. $10 \%$ der deutschen Firmen haben eine Mitarbeiter_in mit nur geringen Dänischkenntnissen, $4 \%$ haben zwei, drei $5 \%, 8 \%$ mehr als drei und $17 \%$ haben „weiß ich nicht“" angekreuzt. $74 \%$ der deutschen Firmen haben keine Mitarbeiter_in, die dänischsprachige E-Mails verfassen kann, $10 \%$ eine, $5 \%$ zwei, $4 \%$ drei und $2 \%$ mehr als drei. Es ist unverkennbar, dass in deutschen Firmen nur wenige Kenntnisse der Sprache des Nachbarlandes Dänemark vorhanden sind. Je höher die abgefragte Zahl an Mitarbeitern mit mehr 
als besseren Dänischkenntnissen und an Mitarbeitern, die dänischsprachige E-Mails schreiben können, umso niedriger fallen die Prozentzahlen aus.

Über wesentlich mehr Kenntnisse der Sprache des Nachbarlandes können dänische Firmen verfügen. Das zeigen auch die proportionalen Verteilungen der Mitarbeiter_innen mit mehr als geringen Deutschkenntnissen und der Mitarbeiter_innen, die deutschsprachige E-Mails beantworten können. Diese Verteilungen haben sich im Vergleich zu Deutschland fast umgedreht. Für die Mitarbeiter_innen mit besseren als geringen Deutschkenntnissen entfallen auf die Zahl eins $7 \%$, auf die Zwei $23 \%$, auf die Drei $15 \%$ und auf „mehr als drei“ $36 \%$. Für die Mitarbeiter_innen, die deutschsprachige E-Mails schreiben können, entfallen auf die Eins $13 \%$, auf die Zwei 18 \%, auf die Drei $17 \%$ und auf ,mehr als drei“ $33 \%$.

\subsubsection{Deutsch- bzw. Dänischgebrauch der Firmenangestellten}

Wiederum werden nur die Unternehmen, die regelmäßige bis sehr häufige Geschäftskontakte mit Dänemark bzw. Deutschland unterhalten, berücksichtigt. Die Prozentzahlen des Sprachgebrauchs beziehen sich aber auf den seltenen bis (fast) ständigen Gebrauch der betreffenden Sprache. Im Fragebogen wurde die Verwendungshäufigkeit der deutschen bzw. dänischen Sprache in vier Abschnitte unterteilt ,,(fast) immer“, „regelmäßig“, „,selten“ und „nie“.

Von den 189 betreffenden deutschen Firmen verwenden im Umgang mit dänischen Unternehmen $26 \%$ mindestens selten deren Landessprache. Für die deutschen Firmen tritt - was den Dänischgebrauch betrifft - folgende Verteilung zutage:

$12 \%$ selten Dänisch

$9 \%$ regelmäßig Dänisch

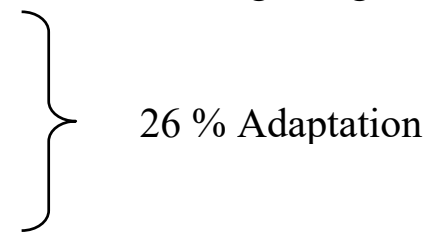

$5 \%$ (fast) immer Dänisch

Die meisten deutschen Unternehmen verwenden Deutsch oder Englisch im Kontakt mit Dänemark:

$30 \%$ selten Deutsch

$42 \%$ regelmäßig Deutsch

$22 \%$ (fast) immer Deutsch

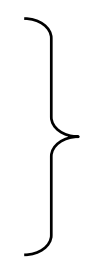

$94 \%$ Nicht-Adaptation

$20 \%$ selten Englisch

$37 \%$ regelmäßig Englisch

38 \% (fast) immer Englisch

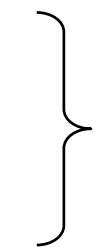

$95 \%$ Englisch als Lingua franca

Die meisten der 117 berücksichtigten dänischen Firmen verwenden Deutsch oder Englisch im Umgang mit deutschen Unternehmen.

$13 \%$ selten Deutsch

$34 \%$ regelmäßig Deutsch

$49 \%$ (fast) immer Deutsch

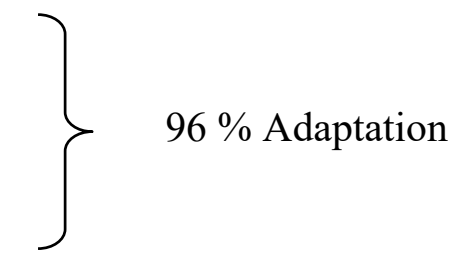

ISSN $1615-3014$ 
$17 \%$ selten Dänisch

$4 \%$ regelmäßig Dänisch

$1 \%$ (fast) immer Dänisch

$22 \%$ selten Englisch

$48 \%$ regelmäßig Englisch

$22 \%$ (fast) immer Englisch

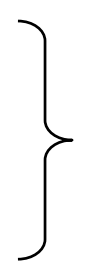

$52 \%$ Nicht-Adaptation

$92 \%$ Englisch als Lingua franca

\subsubsection{Bedarf der Firmenangestellten an Deutsch- bzw. Dänischkenntnissen}

Die Teilnehmer an der Untersuchung wurden mit der Frage „Wie oft wird Ihre Firma Ihrer Meinung nach in den nächsten Jahren Deutsch- bzw. Dänischkenntnisse benötigen?“" aufgefordert, den Deutsch- bzw. Dänischbedarf ihrer Firmen einzuschätzen. Sie konnten eine von vier Antwortkategorien wählen: nie, selten, regelmäßig oder (fast) immer.

Nur $12 \%$ der deutschen Firmen mit mindestens regelmäßigen Geschäftsbeziehungen zu Dänemark teilen mit, einen regelmäßigen Bedarf an Dänischkenntnissen zu haben $(6 \%$ einen sehr häufigen, $32 \%$ einen seltenen und $50 \%$ keinen). Der subjektive Bedarf deckt sich also nicht mit dem objektiven Bedarf (100\%). Deutsche Manager_innen sind sich - gemessen an den deutsch-dänischen Geschäftskontakten - zu wenig ihres Dänischbedarfs bewusst. Ein vergleichbares Defizit lässt sich auf dänischer Seite nicht beobachten: der dänische unbewusste Bedarf an Deutschkenntnissen ist kleiner als $5 \% .15 \%$ der dänischen Firmen mit mindestens regelmäßigen Kontakten zu Deutschland sehen einen seltenen Deutschbedarf, 44 \% einen regelmäßigen und $38 \%$ einen sehr häufigen.

Die Antworten auf die zweite Frage nach dem subjektiven Bedarf zeigen einen subjektiven Deutschbedarf bei $96 \%$ der dänischen Unternehmen, die regelmäßig bis sehr oft mit deutschen Firmen kommunizieren, und einen subjektiven Dänischbedarf bei $44 \%$ der deutschen Firmen. Nachdem geklärt wurde, welche Deutsch- bzw. Dänischkenntnisse die befragten Unternehmen laut Aussage ihrer Manager_innen benötigen, wenden wir uns nun der Frage zu, inwiefern die Manager_innen diesen Bedarf als ungedeckt betrachten. 32\% der Dänen sehen einen Mehrbedarf an Deutschkenntnissen und 25 \% der Deutschen einen Mehrbedarf an Dänischkenntnissen.

Von den dänischen Firmen, die ihrer Meinung nach über keine oder unzureichende Deutschkenntnisse verfügen, während sie einen Bedarf an Deutschkenntnissen bestätigen, haben $76 \%$ mindestens eine Mitarbeiter_in mit besseren als geringen Deutschkenntnissen und $60 \%$ mindestens eine mit geringen Deutschkenntnissen. Die Vergleichszahlen für Deutschland: $21 \%$ und $36 \%$. Wie bei den dänischen Hotels zeigen sich auch hier die hohen Ansprüche der dänischen Manager_innen an das Niveau der Deutschkenntnisse ihrer Mitarbeiter_innen.

Untersuchen wir noch den objektiven Deutschmehrbedarf bzw. objektiven Dänischmehrbedarf. Die Prozentzahl für Deutschland ist alarmierend: $87 \%$ haben einen objektiven Dänischmehrbedarf, d. h. sie stehen regelmäßig bis sehr oft in Verbindung mit dänischen Firmen und verwenden selten oder nie Dänisch. Die Vergleichszahl für Dänemark: $25 \%$ der däni- 
schen Firmen mit mindestens regelmäßigen Geschäftskontakten zu deutschen Firmen verwenden nie oder selten Deutsch.

\subsubsection{Wissen der Firmenangestellten um die deutsch bzw. dänische Mentalität}

Die vorliegende Untersuchung beschäftigt sich nicht nur mit (dem Bedarf an) Deutsch- bzw. Dänischkenntnissen, sondern auch mit (dem Bedarf an) Wissen um die deutsche bzw. dänische Mentalität. So wird untersucht, wie viel Wissen um die deutsche bzw. dänische Mentalität in den befragten Firmen vorhanden ist. Es zeigt sich, dass $81 \%$ der dänischen Unternehmen mit geschäftlichen Kontakten zu Deutschland meinen, Wissen um die deutsche Mentalität zu haben. Ca. 56 \% der deutschen Manager_innen nehmen an, dass ihr Unternehmen Wissen über die dänische Mentalität hat (34\% antworteten „eher nicht“ und $10 \%$,weiß ich nicht"). Es scheint also mehr Wissen um die deutsche Kultur in dänischen Firmen vorhanden zu sein als Wissen um die dänische Kultur in deutschen.

\subsubsection{Bedarf der Firmenangestellten an Wissen über die deutsche bzw. dänische Men- talität}

In der linken Spalte der untenstehenden Tabelle sieht man die genauen Prozentzahlen, welche die Antworten der Firmenmanager_innen in Schleswig-Holstein und Hamburg auf die Frage nach dem Fremdkulturbedarf ergeben haben. In der rechten Spalte sind die entsprechenden Prozentzahlen für Dänemark angegeben.

\begin{tabular}{|l|l|l|}
\hline $\begin{array}{l}\text { Wie viel Wissen über die } \\
\text { dänische bzw. deutsche Men- } \\
\text { talität benötigt Ihre Firma? }\end{array}$ & $\begin{array}{l}\text { in Schleswig-Holstein und } \\
\text { Hamburg }\end{array}$ & in Dänemark \\
\hline kein Wissen & $6,5 \%$ & $2 \%$ \\
\hline sehr wenig & $6,5 \%$ & $3 \%$ \\
\hline eher wenig & $36 \%$ & $11 \%$ \\
\hline teils wenig / teils viel & $27 \%$ & $28 \%$ \\
\hline eher viel & $19 \%$ & $42 \%$ \\
\hline sehr viel & $5 \%$ & $14 \%$ \\
\hline & $100 \%$ & $100 \%$ \\
\hline
\end{tabular}

Tabelle 9: Bedarf an Wissen der Firmenangestellten um die dänische bzw. deutsche Kultur nach Aussage der Manager_innen

Beginnen wir mit der Erläuterung der ersten Spalte der Tabelle zum subjektiven Kulturbedarf. $6,5 \%$ der Firmen in Schleswig-Holstein und Hamburg, die mindestens regelmäßig Geschäftskontakte zu Dänemark haben, geben an, kein Wissen um die dänische Mentalität zu benötigen (6,5\% sehr wenig, $36 \%$ eher wenig, $27 \%$ teils wenig/teils viel, $19 \%$ eher viel und $5 \%$ sehr viel). Schauen wir uns auch die Prozentzahlen der zweiten Spalte mit dem von dänischen Firmen mitgeteilten Bedarf an Wissen um die deutsche Kultur an. Nur $2 \%$ behaupten, dieses Wissen nicht zu benötigen, $3 \%$ sehr wenig zu benötigen, $11 \%$ eher wenig, $28 \%$ teils wenig/teils viel, $42 \%$ eher viel und $14 \%$ sehr viel. Die dänischen Firmenmanager_innen se- 
hen einen viel größeren Bedarf an Kulturwissen als die dänischen Hotelmanager_innen. 56 \% der dänischen Firmenmanager_innen sehen einen (sehr) großen Bedarf, während nur $22 \%$ der dänischen Hotelmanager_innen einen großen bis sehr großen Bedarf bestätigen. Es gibt dagegen keinen Unterschied zwischen den Meinungen deutscher Hotel- und Firmenmanager_innen bezüglich des Fremdkulturbedarfs. Aus dem präsentierten Datenmaterial lässt sich hier und bei den Hotels ersehen, dass deutschen Manager_innen Kulturkenntnisse wichtiger als Sprachkenntnisse sind. Sprachwissen und Kulturwissen sind den dänischen Führungskräften gleich wichtig.

Der subjektive Bedarf wurde noch anhand der Antworten auf eine andere Frage quantifiziert (die Prozentzahlen beziehen sich wiederum nur auf Unternehmen, die mindestens regelmäßig mit Deutschen bzw. Dänen kommunizieren). $13 \%$ der deutschen Unternehmen haben die Antwort „das Unternehmen hat keinen Bedarf an Wissen über die dänische Mentalität“ angekreuzt. Diese haben einen unbewussten Bedarf. $87 \%$ der deutschen Unternehmen haben somit einen subjektiven Bedarf. Einen subjektiven Mehrbedarf haben $30 \%$. Diese haben die Antwort „die Mitarbeiter_innen haben eher zu wenig Dänischkenntnisse“ angekreuzt. $45 \%$ betrachten ihren Bedarf als gedeckt und $12 \%$ haben auf diese Frage (,Haben die Mitarbeiter_innen, die bei den Geschäften mit dänischen Unternehmen mitwirken, Ihrer Meinung nach genügend Wissen über die dänische Mentalität?“) ,,weiß ich nicht“" angekreuzt. Nur $2 \%$ der dänischen Unternehmen haben einen unbewussten Bedarf an Wissen um die deutsche Mentalität. $31 \%$ betrachten ihren Bedarf als nicht gedeckt.

\section{Zusammenfassung und Schlussfolgerungen}

Es werden im Folgenden die Dänischkenntnisse, der Gebrauch der dänischen, deutschen und englischen Sprache sowie der Bedarf an Dänischkenntnissen und der Bedarf an Wissen um die dänische Kultur deutscher Hotels und Firmen quantifiziert. Parallel dazu werden die Deutschkenntnisse und der Deutsch-, Dänisch- und Englischgebrauch dänischer Hotels/Firmen im Kontakt mit deutschen Gästen/Geschäftspartner_innen sowie ihr Bedarf an Deutschkenntnissen und an Wissen über die deutsche Mentalität kurz beschrieben.

Die Resultate belegen, dass die befragten norddeutschen Führungskräfte mit regelmäßigen bis sehr häufigen Kontakten zu Dänen laut eigener Aussage sehr wenige Dänischkenntnisse haben. Nur ca. $10 \%$ verfügen über bessere als geringe Dänischkenntnisse. In ca. $30 \%$ der Hotels, die regelmäßig bis sehr häufig dänische Gäste empfangen, arbeitet mindestens eine Mitarbeiter_in mit besseren als geringen Dänischkenntnissen und in ca. $25 \%$ der Firmen, die regelmäßig bis sehr häufig Geschäftsbeziehungen zu Dänemark haben. In erster Linie machen deutsche Firmen jedoch Gebrauch von den in den dänischen Unternehmen vorhandenen Sprachkenntnissen, indem sie im Kontakt mit diesen Unternehmen häufig Englisch oder Deutsch benutzen. Die Adaptationsstrategie (die sprachliche Anpassung) verfolgen nur ca. $5 \%$ der deutschen Führungskräfte und Angestellten (fast) immer.

Eine Beschreibung des Fremdsprachengebrauchs von Unternehmen ergibt erst Sinn, wenn die Zulänglichkeit bzw. Unzulänglichkeit des festgestellten Zustands angesprochen wird. Bei der Bewertung des festgestellten Zustands ist es erforderlich sich mit dem Fremdsprachenbedarf auseinanderzusetzen. Ca. 45 bis $50 \%$ der deutschen Hotels und Firmen benötigen laut Aussage ihrer Führungskräfte Dänischkenntnisse. Es zeigt sich, dass die Manager_innen für sich 
selbst einen etwas größeren Dänischbedarf sehen (ca. 60 \%). Für nicht gedeckt halten ca. 40 bis $50 \%$ diesen Bedarf. Ca. $45 \%$ der Hoteldirektionen betrachten die Dänischkenntnisse ihres Personals als unzureichend, während nur ca. $25 \%$ der Firmenmanager_innen die unzureichenden Dänischkenntnisse ihrer Angestellten betonen. Somit sehen weniger Firmenmanager_innen als Hotelmanager_innen einen Dänischmehrbedarf für ihre Angestellten. Einen unbewussten Dänischbedarf haben ca. 40 \% der Führungskräfte. Sie geben an, keine Dänischkenntnisse zu benötigen, obwohl sie regelmäßig bis sehr häufig mit Dänen kommunizieren. Ca. 50 bis $55 \%$ der Führungskräfte behaupten, ihre Angestellten, die mindestens regelmäßige Kontakte zu Dänen haben, benötigen keine Dänischkenntnisse. Von den Hotels und Firmen haben ca. $90 \%$ einen objektiven Dänischmehrbedarf, d. h. sie verwenden selten oder nie Dänisch in der Kommunikation mit Dänen. Tabelle 10 veranschaulicht das norddeutsche Datenmaterial.

\begin{tabular}{|l|c|c|c|c|}
\hline & $\begin{array}{l}\text { Deutsche } \\
\text { Hotel- } \\
\text { manager(innen) }\end{array}$ & $\begin{array}{l}\text { Deutsche } \\
\text { Firmen- } \\
\text { manager(innen) }\end{array}$ & $\begin{array}{l}\text { Deutsche } \\
\text { Hotel- } \\
\text { angestellte }\end{array}$ & $\begin{array}{l}\text { Deutsche } \\
\text { Firmen- } \\
\text { angestellte }\end{array}$ \\
\hline $\begin{array}{l}\text { Subjektiver Dänischbe- } \\
\text { darf }\end{array}$ & $60 \%$ & $59 \%$ & $51 \%$ & $44 \%$ \\
\hline $\begin{array}{l}\text { nach Aussage der Füh- } \\
\text { rungskräfte gedeckter } \\
\text { Dänischbedarf }\end{array}$ & $9 \%$ & $19 \%$ & $8 \%$ & $19 \%$ \\
\hline $\begin{array}{l}\text { ungedeckter Dänischbe- } \\
\text { darf } \\
\text { (= subjektiver Dänisch- } \\
\text { mehrbedarf) }\end{array}$ & $51 \%$ & $40 \%$ & $43 \%$ & $25 \%$ \\
\hline $\begin{array}{l}\text { unbewusster Dänisch- } \\
\text { bedarf }\end{array}$ & $40 \%$ & $81 \%$ & $49 \%$ & $56 \%$ \\
\hline $\begin{array}{l}\text { objektiver Dänisch- } \\
\text { mehrbedarf }\end{array}$ & $92 \%$ & $90 \%$ & $87 \%$ \\
\hline
\end{tabular}

Tabelle 10: Dänischbedarf deutscher Unternehmen

Weil die meisten Unternehmen unmöglich die Sprachen all ihrer Gesprächspartner_innen lernen können, erscheint es logisch, sich in der Wahl der zu lernenden Sprachen nach den Frequenzen der Geschäftsbeziehungen sowie nach den Fremdsprachenkenntnissen und den sprachlichen Erwartungen der Gesprächspartner_innen zu richten. Länder wie Dänemark haben sich mehr oder weniger damit abgefunden, dass sehr wenige Ausländer ihre Sprache lernen. Sollten in einem Unternehmen dennoch Dänischkenntnisse vorhanden sein, so könnte es dadurch wichtige Punkte für sich gewinnen. Die hier präsentierten Daten zu Dänischkenntnissen und -gebrauch norddeutscher Unternehmen mit mindestens regelmäßigen Kontakten zu Dänen bringen eindeutig größere Defizite ans Licht, auch aus Sicht der Hälfte der norddeutschen Befragten. Sogar wenn alle Faktoren, von denen es abhängt, welche Sprache die Mitarbeiter_innen deutscher Unternehmen im Kontakt mit Dänen verwenden, berücksichtigt wer- 
den, so kann nur das Fazit gezogen werden, dass sprachenpolitische Maßnahmen in Norddeutschland erforderlich sind.

Was das Kulturwissen betrifft, sind sprachenpolitische Maßnahmen sogar dringend erforderlich. Der subjektive Bedarf an Kulturwissen, den die befragten norddeutschen Unternehmen sehen, ist nämlich viel höher als der subjektive Bedarf an Sprachwissen. Über $80 \%$ haben einen subjektiven Bedarf an Wissen um die dänische Mentalität. Mindestens $30 \%$ der deutschen Firmen und mindestens $50 \%$ der deutschen Hotels halten ihren Bedarf an Wissen um die dänische Mentalität für nicht gedeckt. Die Hotels halten sowohl ihren Bedarf an Sprachals auch an Kulturwissen eher für ungedeckt als die deutschen Firmen.

Kommen wir jetzt zu den dänischen Daten für Hotels und Firmen mit mindestens regelmäßigen Kontakten zu Deutschen. Ca. 80\% der Hotel- und Firmenmanager_innen haben bessere als geringe Deutschkenntnisse. In ca. $60 \%$ der dänischen Hotels ist mindestens eine Mitarbeiter_in mit besseren als geringen Deutschkenntnissen angestellt und in ca. $80 \%$ der Firmen. Ca. $55 \%$ der Hotelmanager_innen und ca. $45 \%$ der Firmenmanager_innen verwenden (fast) immer Deutsch, wenn sie mit Deutschen kommunizieren. Laut Aussage der befragten Führungskräfte benutzen ca. $25 \%$ der Hotelangestellten (fast) immer Deutsch in der Kommunikation mit Deutschen und ca. 50 \% der Firmenangestellten. Außerdem scheinen die dänischen Hotelmanager_innen mehr Deutsch als ihre Angestellten mit Gästekontakt zu verwenden, die Firmenmanager_innen etwas weniger als ihre Mitarbeiter_innen mit Auslandskontakten. Die dänischen Firmenmitarbeiter_innen benutzen häufiger Deutsch als das Hotelpersonal.

Schauen wir uns noch den in Tabelle 11 dargestellten Deutschbedarf der befragen dänischen Unternehmen an.

\begin{tabular}{|l|c|c|c|c|}
\hline & $\begin{array}{l}\text { Dänische } \\
\text { Hotel- } \\
\text { manager(innen) }\end{array}$ & $\begin{array}{l}\text { Dänische } \\
\text { Firmen- } \\
\text { manager(innen) }\end{array}$ & $\begin{array}{l}\text { Dänische } \\
\text { Hotel- } \\
\text { angestellte }\end{array}$ & $\begin{array}{l}\text { Dänische } \\
\text { Firmen- } \\
\text { angestellte }\end{array}$ \\
\hline $\begin{array}{l}\text { Subjektiver Deutschbe- } \\
\text { darf }\end{array}$ & $95 \%$ & $96 \%$ & $90 \%$ & $96 \%$ \\
\hline $\begin{array}{l}\text { nach Aussage der Füh- } \\
\text { rungskräfte gedeckter } \\
\text { Deutschbedarf }\end{array}$ & $73 \%$ & $58 \%$ & $47 \%$ & $64 \%$ \\
\hline $\begin{array}{l}\text { ungedeckter Deutschbe- } \\
\text { darf } \\
(=\text { subjektiver Deutsch- } \\
\text { mehrbedarf })\end{array}$ & $22 \%$ & $38 \%$ & $43 \%$ & $32 \%$ \\
\hline $\begin{array}{l}\text { unbewusster Deutschbe- } \\
\text { darf }\end{array}$ & $5 \%$ & $4 \%$ & $32 \%$ & $25 \%$ \\
\hline $\begin{array}{l}\text { objektiver Deutschmehr- } \\
\text { bedarf }\end{array}$ & $32 \%$ & $37 \%$ & $4 \%$ \\
\hline
\end{tabular}

Tabelle 11: Deutschbedarf dänischer Unternehmen 
Der subjektive Deutschbedarf dänischer Unternehmen ist mit ca. $95 \%$ sehr hoch. Einen subjektiven Deutschmehrbedarf haben ca. $20 \%$ der dänischen Hotelführungskräfte, ca. $40 \%$ der dänischen Firmenmanager_innen, ca. $40 \%$ der dänischen Hotelangestellten und ca. $30 \%$ der dänischen Firmenangestellten. Ein unbewusster Deutschbedarf konnte nur bei ca. $5 \%$ der dänischen Unternehmen festgestellt werden. Der objektive Deutschmehrbedarf ist höher und liegt zwischen 25 und 37 \%. Die Stellung der deutschen Sprache in Dänemark ist eine völlig andere als die des Dänischen in Deutschland. Die befragten dänischen Unternehmen, die regelmäßig bis sehr häufig Geschäftskontakte mit Deutschland unterhalten, verwenden sehr häufig Deutsch. Mindestens jede dritte dänische Manager_in ist dennoch der Meinung, in ihrem Unternehmen sind nicht genug Deutschkenntnisse sowie Kenntnisse um die deutsche Mentalität vorhanden.

Wir müssen Fremdsprachenkenntnisse auch als Schlüssel zur Erschließung (größerer Anteile) ausländischer Märkte betrachten. Es ist im wirtschaftlichen Bereich nicht nur von einem Deutsch- bzw. Dänischbedarf die Rede, wenn schon mindestens regelmäßige Kontakte zwischen Dänen und Deutschen geknüpft wurden. Außerdem können (bessere) Dänischkenntnisse aus seltenen oder regelmäßigen Geschäftskontakten zu Dänen sehr häufige werden lassen oder (noch bessere) Deutschkenntnisse in dänischen Unternehmen können zu (noch mehr) Geschäftsbeziehungen mit Deutschland führen. Höher ist der objektive dänische Deutschbedarf auch schon dadurch, dass alle deutschsprachigen Gäste/Geschäftspartner_innen berücksichtig werden müssen, auch die aus Österreich und der Schweiz.

Die vorliegende Untersuchung hat deutlich gemacht, dass die Stellung des Dänischen in Norddeutschland verbessert werden muss. Die norddeutschen Unternehmen mit einem unbewussten Dänischbedarf müssen sich ihres Bedarfs bewusst werden und die Unternehmen mit einem ungedeckten subjektiven Dänischbedarf diesem Bedarf die ihm gebührende Aufmerksamkeit schenken. Auch die deutschen Bildungspolitiker_innen sind gefordert, den Fremdsprachenunterricht an Schulen und Hochschulen am Dänischbedarf der Unternehmen auszurichten.

Die deutsch-dänischen Geschäftsbeziehungen müssen noch erfolgreicher bewältigt werden. Fehlende Dänischkenntnisse und Kulturunterschiede erschweren die deutsch-dänische Kommunikation in nicht zu vernachlässigendem Maße. Auch die anderen SMiK-Teilprojekte sind zu dem Ergebnis gekommen (cf. z. B. Hofmann/Hallsteinsdóttir und Müller/Hallsteinsdóttir in diesem Heft). Sowohl ein für die einzelnen Wirtschaftszweige maßgeschneidertes Lernen der Landessprache des Nachbarlandes als auch ein optimales interkulturelles Lernen müssen ermöglicht werden (am besten in Kombination miteinander).

Im Rahmen des SMiK-Teilprojekts Interkulturelle Kommunikation, Kulturdimensionen und Stereotype wurde nicht nur eine Bedarfsanalyse durchgeführt, sondern auch empirische Ergebnisse bezüglich der deutschen und dänischen Kulturmerkmale gewonnen. Das Hauptziel des Teilprojekts ist es, diese Ergebnisse zu didaktisieren. Die Ergebnisse der Fragebogenaktion unter deutschen und dänischen Hotel- und Firmenmanager_innen haben ans Licht gebracht, dass diese einen sehr großen Bedarf an der Vermittlung von Wissen über die dänische bzw. deutsche Mentalität sehen. Die Daten zu den deutschen und dänischen Kulturmerkmalen können bei der Vermittlung dieses Wissens gewinnbringend eingesetzt werden. 


\section{Literatur}

Beamer, Linda (1992): "Learning intercultural communication competence”. Journal of Business Communication 29: 285-303.

Beneke, Juergen (1991): „Englisch als lingua franca oder als Medium interkultureller Kommunikation“. In: Grebing, Renate (ed.): Grenzenloses Sprachenlernen. Festschrift für Reinhold Freudenstein. Oxford, Cornelsen/Oxford University Press: 54-66.

Coulmas, Florian (1987): "Why speak English?" In: Knapp, Karlfried/Enninger, Werner/Knapp-Potthoff, Amelie (eds.): Analyzing intercultural communication. Berlin/New York, de Gruyer: 95-107.

Hagen, Stephen (1993): Languages in European business. A regional survey of small \& medium-sized companies. London: City Technology Colleges Trust Limited.

Hofstede, Geert/Hofstede, Gert Jan (1991): Cultures and Organizations. Software of the Mind. Berkshire: Mcgraw-Hill.

Losche, Helga/Püttker, Stephanie (2009): Interkulturelle Kommunikation. Theoretische Einführung und Sammlung praktischer Interaktionsübungen. Augsburg: Ziel.

Rehbein, Jochen (1995): "International sales talk". In: Ehrlich Konrad/Wagner, Johannes (eds.): The discourse of business negotiation. Berlin/New York, de Gruyer: 67-102.

Scholz, Christian/Stein, Volker (2013): Interkulturelle Wettbewerbsstrategien. Göttingen/Bristol: Vandenhoeck \& Ruprecht.

Scholz, Joachim et al. (2015): Marktinformation. Incoming-Tourismus Deutschland 2016. Dänemark. Frankfurt a. M.: Deutsche Zentrale für Tourismus e. V. (DZT). www.germany.travel/media/pdf/marktinformationen_lang_/regionalmanagement_nordost europa/Daenemark.pdf [07.07.2014].

Vandermeeren, Sonja (1998): Fremdsprachen in europäischen Unternehmen. Untersuchungen zu Bestand und Bedarf im Geschäftsalltag mit Empfehlungen für Sprachenpolitik und Sprachunterricht. Waldsteinberg: Popp.

Vandermeeren, Sonja (2003): "German language needs in Danish companies". Hermes, Journal of Linguistics 31: 13-29.

Vandermeeren, Sonja (2005): "Foreign Language Needs of Business Firms". In: Long, Michael (ed.): Second Language Needs Analysis. Cambridge, Cambridge University Press: 169-181.

Vandermeeren, Sonja (2013): „Kulturdimensionen und Stereotype: eine empirische Untersuchung in Dänemark und Deutschland“. In: Schneider-Wiejowski, Karina/KellmeierRehbein, Birte/ Haselhuber, Jacob (eds.): Vielfalt, Variation und Stellung der deutschen Sprache. Festschrift für Ulrich Ammon. Berlin/Bristol, de Gruyter: 563-578.

Vandermeeren, Sonja (2016): „Kulturdimensionen und Stereotype. Begriffserläuterungen und -operationalisierungen“. In: Hallsteinsdóttir, Erla et al. (eds.): Perspektiven der Stereotypenforschung. Frankfurt a. M. etc., Lang: 163-183.

Vandermeeren, Sonja/Hofmann, Annika (2016): „Kulturdimensionen und Stereotype: Ergebnisse einer Fragebogenuntersuchung mit Danfoss-Mitarbeiter_innen“. In: Hallsteinsdóttir, Erla et al. (eds.): Perspektiven der Stereotypenforschung. Frankfurt a. M. etc., Lang: 185206. 